Yoel Feler

\title{
Spaces of geometrically generic configurations
}

Received December 7, 2005 and in revised form March 19, 2007

\begin{abstract}
Let $X$ denote either $\mathbb{C P}^{m}$ or $\mathbb{C}^{m}$. We study certain analytic properties of the space $\mathcal{E}^{n}(X, g p)$ of ordered geometrically generic $n$-point configurations in $X$. This space consists of all $q=\left(q_{1}, \ldots, q_{n}\right) \in X^{n}$ such that no $m+1$ of the points $q_{1}, \ldots, q_{n}$ belong to a hyperplane in $X$. In particular, we show that for large enough $n$ any holomorphic map $f: \mathcal{E}^{n}\left(\mathbb{C P}^{m}, g p\right) \rightarrow$ $\mathcal{E}^{n}\left(\mathbb{C P}^{m}, g p\right)$ commuting with the natural action of the symmetric group $\mathbf{S}(n)$ in $\mathcal{E}^{n}\left(\mathbb{C} \mathbb{P}^{m}, g p\right)$ is of the form $f(q)=\tau(q) q=\left(\tau(q) q_{1}, \ldots, \tau(q) q_{n}\right), q \in \mathcal{E}^{n}\left(\mathbb{C P}^{m}, g p\right)$, where $\tau: \mathcal{E}^{n}\left(\mathbb{C P}^{m}, g p\right) \rightarrow$ $\operatorname{PSL}(m+1, \mathbb{C})$ is an $\mathbf{S}(n)$-invariant holomorphic map. A similar result holds true for mappings of the configuration space $\mathcal{E}^{n}\left(\mathbb{C}^{m}, g p\right)$.
\end{abstract}

Keywords. Configuration space, geometrically generic configurations, vector braids, points in general position, holomorphic endomorphism

\section{Introduction}

In this paper we study certain analytic properties of the spaces of geometrically generic point configurations in projective and affine spaces.

The most traditional configuration space $\mathcal{C}^{n}=\mathcal{C}^{n}(X)$ of a complex space $X$ consists of all $n$-point subsets ("configurations") $Q=\left\{q_{1}, \ldots, q_{n}\right\} \subset X$. If $X$ carries an additional geometric structure, it may be taken into account. Say if $X$ is either the projective space $\mathbb{C P}^{m}$ or the affine space $\mathbb{C}^{m}$ and $n>m$ then the space $\mathcal{C}^{n}=\mathcal{C}^{n}(X, g p)$ of geometrically generic configurations consists of all $n$-point subsets $Q \subset X$ such that no hyperplane in $X$ contains more than $m$ points of $Q$. The corresponding ordered configuration space $\mathcal{E}^{n}=$ $\mathcal{E}^{n}(X, g p)$ consists of all $q=\left(q_{1}, \ldots, q_{n}\right) \in X^{n}$ such that the set $Q=\left\{q_{1}, \ldots, q_{n}\right\} \subset X$ belongs to $\mathcal{C}^{n}(X, g p)$. The left action of the symmetric group $\mathbf{S}(n)$ on $\mathcal{E}^{n}$ defined by $\sigma\left(q_{1}, \ldots, q_{n}\right)=\left(q_{\sigma^{-1}(1)}, \ldots, q_{\sigma^{-1}(n)}\right)$ is free; the corresponding orbit map identifies with the tautological covering map $p: \mathcal{E}^{n} \ni\left(q_{1}, \ldots, q_{n}\right) \mapsto\left\{q_{1}, \ldots, q_{n}\right\} \in \mathcal{C}^{n}$, which is an unbranched Galois covering with Galois group $\mathbf{S}(n)$.

Let Aut $_{g} X$ be the subgroup of the holomorphic automorphism group Aut $X$ of $X$ consisting of all elements $A \in$ Aut $X$ that respect the geometrical structure of $X$; i.e. for $X=\mathbb{C P}^{m}$ we have Aut ${ }_{g} X=\operatorname{PSL}(m+1, \mathbb{C})=$ Aut $X$, whereas for $X=\mathbb{C}^{m}$ it is known

Y. Feler: Department of Mathematics, Weizmann Institute of Science, Rehovot, Israel 76100; e-mail: Yoel.Feler@weizmann.ac.il

Mathematics Subject Classification (2000): 14J50, 32H25, 32H02, 32M99 
that $\operatorname{Aut}_{g} X=\mathbf{A f f}(m, \mathbb{C}) \subsetneq \operatorname{Aut}\left(\mathbb{C}^{m}\right)$. Notice that in both cases $\operatorname{Aut}_{g} X$ is a complex Lie group. The Aut ${ }_{g} X$ action in $X$ induces the diagonal Aut ${ }_{g} X$ actions in $\mathcal{C}^{n}$ and $\mathcal{E}^{n}$ defined by $A Q:=\left\{A q_{1}, \ldots, A q_{n}\right\}$ for all $Q=\left\{q_{1}, \ldots, q_{n}\right\} \in \mathcal{C}^{n}$ and $A q:=\left(A q_{1}, \ldots, A q_{n}\right)$ for all $q=\left(q_{1}, \ldots, q_{n}\right) \in \mathcal{E}^{n}$.

Definition 1.1. An endomorphism $F$ of $\mathcal{C}^{n}$ is said to be tame if there is a holomorphic map $T: \mathcal{C}^{n} \rightarrow$ Aut $_{g} X$ such that $F(Q)=F_{T}(Q):=T(Q) Q$ for all $Q \in \mathcal{C}^{n}$. Similarly, an endomorphism $f$ of $\mathcal{E}^{n}$ is called tame if there is an $\mathbf{S}(n)$-invariant holomorphic map $\tau: \mathcal{E}^{n} \rightarrow$ Aut $_{g} X$ and $\sigma \in \mathbf{S}(n)$ such that $f(q)=f_{\tau, \sigma}(q):=\sigma \tau(q) q$ for all $q \in \mathcal{E}^{n}$.

When $X=\mathbb{C}^{m}$, an endomorphism $F$ of $\mathcal{C}^{n}\left(\mathbb{C}^{m}, g p\right)$ is said to be quasitame if there is a holomorphic map $T: \mathcal{C}^{n}\left(\mathbb{C}^{m}, g p\right) \rightarrow \operatorname{PSL}(m+1, \mathbb{C})$ such that $F(Q)=$ $F_{T}(Q):=T(Q) Q$ for all $Q \in \mathcal{C}^{n}\left(\mathbb{C}^{m}, g p\right)$; notice that the latter condition implies that $T(Q) Q \subset \mathbb{C}^{m}$ for any $Q \in \mathcal{C}^{n}\left(\mathbb{C}^{m}\right.$, gp). Similarly, an endomorphism $f$ of $\mathcal{E}^{n}=\mathcal{E}^{n}\left(\mathbb{C}^{m}, g p\right)$ is quasitame if there is an $\mathbf{S}(n)$-invariant holomorphic map $\tau: \mathcal{E}^{n}\left(\mathbb{C}^{m}, g p\right) \rightarrow \operatorname{PSL}(m+1, \mathbb{C})$ and $\sigma \in \mathbf{S}(n)$ such that $\tau(q) q_{1}, \ldots, \tau(q) q_{n} \in \mathbb{C}^{m}$ and $f(q)=\sigma \tau(q) q$ for any $q=\left(q_{1}, \ldots, q_{n}\right) \in \mathcal{E}^{n}\left(\mathbb{C}^{m}, g p\right)$.

The left $\mathbf{S}(n)$-action on $\mathcal{E}^{n}(X, g p)$ induces the left $\mathbf{S}(n)$-action on the set of all maps $f: \mathcal{E}^{n}(X, g p) \rightarrow \mathcal{E}^{n}(X, g p)$ defined by $\sigma f=\sigma\left(f_{1}, \ldots, f_{n}\right)=\left(f_{\sigma^{-1}(1)}, \ldots, f_{\sigma^{-1}(n)}\right)$ for $f=\left(f_{1}, \ldots, f_{n}\right): \mathcal{E}^{n}(X, g p) \rightarrow \mathcal{E}^{n}(X, g p)$ and $\sigma \in \mathbf{S}(n)$.

Definition 1.2. A continuous map $f: \mathcal{E}^{n} \rightarrow \mathcal{E}^{n}$ is called equivariant if there is $\alpha \in$ Aut $\mathbf{S}(n)$ such that $f(\sigma q)=\alpha(\sigma) f(q)$ for all $q \in \mathcal{E}^{n}$ and $\sigma \in \mathbf{S}(n)$. (This property is slightly weaker than the "standard" equivariance, which requires that $f(\sigma q)=\sigma f(q)$.)

When $m=1$, that is, when $X=\mathbb{C P}^{1}$ or $\mathbb{C}^{1}$, the spaces of geometrically generic configurations in $X$ coincide with the usual configuration spaces $\mathcal{C}^{n}(X)$ and $\mathcal{E}^{n}(X)$. V. Lin [4] 6] proved that when $n>4$ and $X$ is $\mathbb{C}$ or $\mathbb{C P}^{1}$, any equivariant endomorphism $F$ of $\mathcal{E}^{n}(X)$ is tame. (He also completely described all endomorphisms of the spaces $\mathcal{C}^{n}\left(\mathbb{C P}^{1}\right)$ and $\mathcal{C}^{n}(\mathbb{C})$; moreover, thanks to the works of V. Zinde [11- -15$]$ and the author [3], such a description is known for endomorphisms of traditional configuration spaces of all nonhyperbolic algebraic curves.)

In this paper we treat the equivariant endomorphisms of $\mathcal{E}^{n}\left(\mathbb{C P}^{m}, g p\right)$ and $\mathcal{E}^{n}\left(\mathbb{C}^{m}, g p\right)$. The following theorem contains the main results of this work.

Theorem 1.3. Let $m>1, n \geq m+3$ and $n \neq 2 m+2$.

(a) Any equivariant endomorphism $f$ of $\mathcal{E}^{n}\left(\mathbb{C P}^{m}, g p\right)$ is tame.

(b) Any equivariant endomorphism $f$ of $\mathcal{E}^{n}\left(\mathbb{C}^{m}, g p\right)$ is quasitame.

In more detail, for any equivariant endomorphism $f$ of $\mathcal{E}^{n}$, there is an $\mathbf{S}(n)$-invariant holomorphic map $\tau: \mathcal{E}^{n} \rightarrow \operatorname{PSL}(m+1, \mathbb{C})$ and $\sigma \in \mathbf{S}(n)$ such that

$$
f(q)=\sigma \tau(q) q=\left(\tau(q) q_{\sigma^{-1}(1)}, \ldots, \tau(q) q_{\sigma^{-1}(n)}\right) \quad \text { for all } q=\left(q_{1}, \ldots, q_{n}\right) \in \mathcal{E}^{n} .
$$

Remark 1.4. Any tame endomorphism $f=f_{\tau, \sigma}$ of $\mathcal{E}^{n}$ is equivariant; the corresponding automorphism $\alpha \in \operatorname{Aut} \mathbf{S}(n)$ is just the automorphism $s \mapsto \sigma s \sigma^{-1}$. The same holds true for quasitame maps. 
Remark 1.5. Theorem 1.3 b) is not complete, since at the moment I do not know whether there are endomorphisms of $\mathcal{E}^{n}\left(\mathbb{C}^{m}, g p\right)$ that are quasitame but not tame. Moreover, although I think that Theorem 1.3 holds true for $n=2 m+2$, in this case I could not overcome some technical difficulties which arose in the proof.

The plan of the proof is as follows. Let $X=\mathbb{C}^{m}$ or $\mathbb{C} P^{m}$. To study an equivariant endomorphism $f$ of $\mathcal{E}^{n}=\mathcal{E}^{n}(X, g p)$, we start with an explicit description of all nonconstant holomorphic maps $\lambda: \mathcal{E}^{n} \rightarrow \mathbb{C} \backslash\{0,1\}$ (cf. [6], [12], [13] and [3]). The set $L\left(\mathcal{E}^{n}\right)$ of all such maps is finite and separates points of a certain submanifold $M \subset \mathcal{E}^{n}$ of complex codimension $m(m+1)$; we endow $L=L\left(\mathcal{E}^{n}\right)$ with a special simplicial structure. The action of $\mathbf{S}(n)$ in $\mathcal{E}^{n}$ induces a simplicial $\mathbf{S}(n)$-action in the complex $L$. An endomorphism $f$ induces a simplicial self-map $f^{*}$ of $L$ via $f^{*}: L \ni \lambda \mapsto f^{*} \lambda=$ $\lambda \circ f \in L$, which carries important information about $f$. Since $f$ is equivariant, $f^{*}$ is nicely related to the $\mathbf{S}(n)$-action on $L$. Studying all these things together, we come to the desired conclusion.

Remark 1.6. The topology of the spaces in question is of great independent interest. A. I. Barvinok calculated the first homology group of the ordered space $\mathcal{E}^{n}\left(\mathbb{C}^{2}, g p\right.$ ) (see [1]). V. Moulton [8] found the generators and some generating relations of the fundamental groups $\pi_{1}\left(\mathcal{E}^{n}\left(\mathbb{C}^{m}, g p\right)\right)$ and $\pi_{1}\left(\mathcal{E}^{n}\left(\mathbb{C P}^{m}, g p\right)\right)$. T. Terasoma [9] found a presentation of $\pi_{1}\left(\mathcal{E}^{n}\left(\mathbb{C P}^{m}, g p\right)\right)$ for $n>m+1$.

\section{Some properties of generic configurations}

The spaces $\mathcal{E}^{n}$ have the following explicit algebraic description.

Any point $q \in\left(\mathbb{C P}^{m}\right)^{n}$ may be represented as a 'matrix'

$$
q=\left(\begin{array}{c}
q_{1} \\
\vdots \\
q_{n}
\end{array}\right)=\left(\begin{array}{c}
{\left[z_{1,1}: \cdots: z_{1, m+1}\right]} \\
\vdots \\
{\left[z_{n, 1}: \cdots: z_{n, m+1}\right]}
\end{array}\right) \in\left(\mathbb{C P}^{m}\right)^{n},
$$

where $q_{j}=\left[z_{j, 1}: \cdots: z_{j, m+1}\right] \in \mathbb{C P}^{m}, j=1, \ldots, n$. For $m+1$ distinct indices $i_{1}, \ldots, i_{m+1} \in\{1, \ldots, n\}$, the determinant

$$
d_{i_{1}, \ldots, i_{m+1}}(q)=\left|\begin{array}{cccc}
z_{i_{1}, 1} & \ldots & z_{i_{1}, m} & z_{i_{1}, m+1} \\
\vdots & \vdots & \vdots & \vdots \\
z_{i_{m+1}, 1} & \ldots & z_{i_{m+1}, m} & z_{i_{m+1}, m+1}
\end{array}\right|
$$

is a homogeneous polynomial of degree $m+1$ in the homogeneous coordinates $\left[z_{1,1}\right.$ : $\left.\cdots: z_{1, m+1}\right], \ldots,\left[z_{n, 1}: \cdots: z_{n, m+1}\right]$. The space $\mathcal{E}^{n}\left(\mathbb{C P}^{m}, g p\right)$ consists of all $q$ of the form (1) such that $d_{i_{1}, \ldots, i_{m+1}}(q) \neq 0$ for all distinct $i_{1}, \ldots, i_{m+1} \in\{1, \ldots, n\}$.

Similarly, the space $\mathcal{E}^{n}\left(\mathbb{C}^{m}, g p\right)$ consists of all matrices

$$
q=\left(\begin{array}{c}
q_{1} \\
\vdots \\
q_{n}
\end{array}\right)=\left(\begin{array}{c}
z_{1,1}, \ldots, z_{1, m}, 1 \\
\vdots \\
z_{n, 1}, \ldots, z_{n, m}, 1
\end{array}\right) \in\left(\mathbb{C}^{m}\right)^{n}
$$


with all $q_{j}=\left(z_{j, 1}, \ldots, z_{j, m}\right) \in \mathbb{C}^{m}$ such that

$$
d_{i_{1}, \ldots, i_{m+1}}(q)=\left|\begin{array}{cccc}
z_{i_{1}, 1} & \ldots & z_{i_{1}, m} & 1 \\
\vdots & \vdots & \vdots & \vdots \\
z_{i_{m+1}, 1} & \ldots & z_{i_{m+1}, m} & 1
\end{array}\right| \neq 0
$$

for all distinct $i_{1}, \ldots, i_{m+1} \in\{1, \ldots, n\}$.

We refer to the components $q_{1}, \ldots, q_{n}$ of a point $q=\left(q_{1}, \ldots, q_{n}\right)$ in $\mathcal{E}^{n}\left(\mathbb{C P}^{m}, g p\right)$ or in $\mathcal{E}^{n}\left(\mathbb{C}^{m}, g p\right)$ as vector coordinates of $q$.

Although we use the same notation for determinant polynomials in both cases, projective and affine, it will be clear from the context which one we mean.

One can easily verify the following statement.

Lemma 2.1. All minors of the matrices (1) and (2) are irreducible homogeneous polynomials of the entries $z_{t, s}$.

Notation 2.2. By a multiindex we mean an ordered set $\mathbf{i}=\left(i_{1}, \ldots, i_{s}\right)$ with distinct $i_{1}, \ldots, i_{s} \in\{1, \ldots, n\}$. Sometimes we forget the order and write $i \in \mathbf{i}$ and $\# \mathbf{i}=s$. If $s=1$ and $i \in\{1, \ldots, n\}$, we may write $\mathbf{i}=i$ instead of $\mathbf{i}=(i)$.

For $t$ multiindices $\mathbf{i}_{1}=\left(i_{1}^{1}, \ldots, i_{s}^{1}\right), \ldots, \mathbf{i}_{t}=\left(i_{1}^{t}, \ldots, i_{s}^{t}\right)$ such that $i_{1}^{1}, \ldots, i_{s}^{1}, \ldots$,

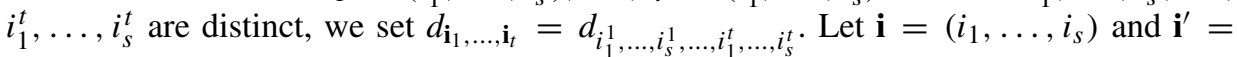
$\left(i_{1}, \ldots, i_{s-1}\right)$; for any $i \in\left\{i_{1}, \ldots, i_{s}\right\}$ and any $k=1, \ldots, s-1$, we denote by $D_{\mathbf{i} ; i, k}$ the $(s-1) \times(s-1)$ minor of the $s \times s$ matrix

$$
Z_{\mathbf{i}}=\left(\begin{array}{c}
z_{i_{1}, 1}, \ldots, z_{i_{1}, s-1}, 1 \\
\vdots \\
z_{i_{s}, 1}, \ldots, z_{i_{s}, s-1}, 1
\end{array}\right)
$$

complementary to $z_{i, k}$; for $i=i_{s}$, we write $\delta_{\mathbf{i}^{\prime} ; k}$ instead of $D_{\mathbf{i} ; i_{s}, k}$. Let $\mathbf{I}^{s}$ denote the set of all multiindices $\mathbf{i}=\left(i_{1}, \ldots, i_{s}\right)$ such that $1 \leq i_{1}<\cdots<i_{s} \leq n$. For $\mathbf{i}=\left(i_{1}, \ldots, i_{s}\right) \in \mathbf{I}^{s}$ and $\mathbf{j}=\left(j_{1}, \ldots, j_{t}\right) \in \mathbf{I}^{t}$, we define the multiindices $\mathbf{i} \cap \mathbf{j}$ and $\mathbf{i} \backslash \mathbf{j}$ in the evident way; if $\mathbf{i} \cap \mathbf{j}=\emptyset$, we define the multiindex $\mathbf{i} \cup \mathbf{j} \in \mathbf{I}^{s+t}$ by an appropriate reordering of the components $i_{1}, \ldots, i_{s}, j_{1}, \ldots, j_{t}$. For $1 \leq j \leq n$, set $\mathbf{I}_{j}^{s}=\left\{\mathbf{i} \in \mathbf{I}^{s} \mid j \notin \mathbf{i}\right\}$.

Now we can establish the following property of the determinant polynomials.

Lemma 2.3. Let $i_{0} \in\{1, \ldots, n\}$, $\mathbf{i}_{0}=\left(i_{1}, \ldots, i_{m}\right) \in \mathbf{I}_{i_{0}}^{m}$ and $L \subset \mathbb{C}^{m n}$ be a linear subspace defined by the relations $z_{i_{1}, 2}=\cdots=z_{i_{m}, 2}$. For $\mathbf{i} \in \mathbf{I}_{i_{0}}^{m+1},\left.\operatorname{deg} d_{\mathbf{i}}\right|_{L}=m$; the polynomial $\left.d_{\mathbf{i}}\right|_{L}$ is irreducible if and only if $\#\left(\mathbf{i} \cap \mathbf{i}_{0}\right)<m$; moreover, if $\#\left(\mathbf{i} \cap \mathbf{i}_{0}\right)=m$, then $\left.d_{\mathbf{i}}\right|_{L}= \pm D_{\mathbf{i} ; i, 2} \cdot\left(z_{i, 2}-z_{i_{1}, 2}\right)$, where $i \in \mathbf{i} \backslash \mathbf{i}_{0}$.

Proof. Assume that $\#\left(\mathbf{i} \cap \mathbf{i}_{0}\right)=m$, thus $\#\left(\mathbf{i} \backslash \mathbf{i}_{0}\right)=1$. Set $(i)=\mathbf{i} \backslash \mathbf{i}_{0}$. By the Lagrange determinant decomposition formula, we can show that $\left.d_{\mathbf{i}}\right|_{L}= \pm D_{\mathbf{i} ; i, 2} \cdot\left(z_{i, 2}-z_{i_{1}, 2}\right)$, i.e. $\left.d_{\mathbf{i}}\right|_{L}$ is reducible and $\left.\operatorname{deg} d_{\mathbf{i}}\right|_{L}=m$. Now assume that $\#\left(\mathbf{i} \cap \mathbf{i}_{0}\right)<m$. Let $i, j \in \mathbf{i} \backslash \mathbf{i}_{0}$ be two distinct indices. It is clear that if we prove that $\left.d_{i, j, i_{2}, \ldots, i_{m}}\right|_{L}$ is irreducible and 
$\left.\operatorname{deg} d_{i, j, i_{2}, \ldots, i_{m}}\right|_{L}=m$, the same statements for $\left.d_{\mathbf{i}}\right|_{L}$ hold true. So set $\mathbf{j}=\left(i, j, i_{2}, \ldots, i_{m}\right)$. Clearly, $\left.d_{\mathbf{j}}\right|_{L}=\left(z_{i_{2}, 2}-z_{i, 2}\right) \cdot D_{\mathbf{j} ; i, 2}-\left(z_{i_{2}, 2}-z_{j, 2}\right) \cdot D_{\mathbf{j} ; j, 2}$ and $\left.\operatorname{deg} d_{\mathbf{j}}\right|_{L}=m$. Therefore, $\left.d_{\mathbf{j}}\right|_{L}$ is a linear function of the variables $z_{i, 2}, z_{j, 2}, z_{i_{2}, 2}$ with coefficients $D_{\mathbf{j} ; i, 2}, D_{\mathbf{j} ; j, 2}, D_{\mathbf{j} ; i, 2}-$ $D_{\mathbf{j} ; i, 2}$. By Lemma 2.1, $D_{\mathbf{j} ; i, 2}, D_{\mathbf{j} ; j, 2}$ are irreducible. Thus $D_{\mathbf{j} ; i, 2}, D_{\mathbf{j} ; j, 2}, D_{\mathbf{j} ; i, 2}-D_{\mathbf{j} ; i, 2}$ are pairwise co-prime. This implies that $\left.d_{\mathbf{j}}\right|_{L}$ is irreducible, and completes the proof.

\subsection{The direct decomposition of $\mathcal{E}^{n}\left(\mathbb{C P}^{m}, g p\right)$}

Here we observe that $\mathcal{E}^{n}\left(\mathbb{C P}^{m}, g p\right)$ admits a natural representation as a Cartesian product of its subspace of codimension $m(m+2)$ and the group PSL $(m+1, \mathbb{C})$.

Definition 2.4. Set $v_{1}=[1: 0: \ldots: 0], v_{2}=[0: 1: 0: \cdots: 0], \ldots, v_{m+1}=$ $[0: \cdots: 0: 1]$ and $w=[1: \cdots: 1]$. The subspace $M_{m, n} \subset \mathcal{E}^{n}\left(\mathbb{C P}^{m}\right.$, gp $)$ defined by $M_{m, n}=\left\{q=\left(q_{1}, \ldots, q_{n}\right) \in \mathcal{E}^{n}\left(\mathbb{C P}^{m}, g p\right) \mid q_{i}=v_{i} \forall i=1, \ldots, m+1, q_{m+2}=w\right\}$ is called the reduced space of geometrically generic ordered configurations.

Lemma 2.5. Let $n \geq m+3$. For every $q \in \mathcal{E}^{n}\left(\mathbb{C P}^{m}\right.$, gp), there is a unique $\gamma(q) \in$ PSL $(m+1)$ such that $\gamma(q) q \in M_{m, n}$. The map $\gamma: \mathcal{E}^{n}\left(\mathbb{C P}^{m}, g p\right) \ni q \mapsto \gamma(q) \in$ $\operatorname{PSL}(m+1)$ is holomorphic.

For $m=1$ the statement of the lemma is common knowledge; the case $m=2$ is treated in [2, Chap. V, Sec. 109, Theorem 36]. Since for $m>2$ the proof is similar to the proof of the case $m=2$, we shall omit it.

Corollary 2.6. The mutually inverse maps $A: \mathcal{E}^{n}\left(\mathbb{C P}^{m}, g p\right) \quad \ni \quad q \mapsto A(q)=$ $(\gamma(q), \gamma(q) q) \in \mathbf{P S L}(m+1) \times M_{m, n}$ and $B: \operatorname{PSL}(m+1) \times M_{m, n} \ni(T, \tilde{q}) \mapsto B(T, \tilde{q})=$ $T^{-1} \tilde{q} \in \mathcal{E}^{n}\left(\mathbb{C P}^{m}\right.$, gp $)$ induce a natural biholomorphic isomorphism $\mathcal{E}^{n}\left(\mathbb{C P}^{m}\right.$, gp $) \cong$ $\operatorname{PSL}(m+1) \times M_{m, n}$.

Remark 2.7. The above corollary implies that $\mathcal{E}^{n}\left(\mathbb{C P}^{m}, g p\right)$ and $\mathcal{C}^{n}\left(\mathbb{C P}^{m}, g p\right)$ are irreducible non-singular affine algebraic varieties, and hence Stein manifolds. Indeed, in the above decomposition of $\mathcal{E}^{n}\left(\mathbb{C P}^{m}, g p\right)$ both PSL $(m+1)$ and $M_{m, n}$ are such varieties and hence $\mathcal{E}^{n}\left(\mathbb{C P} \mathbb{P}^{m}, g p\right)$ is also of the same nature. Since $\mathbf{S}(n)$ is finite and its action on $\mathcal{E}^{n}\left(\mathbb{C P}^{m}, g p\right)$ is free, the same is true for $\mathcal{C}^{n}\left(\mathbb{C P}^{m}, g p\right)$. The same properties hold true for $\mathcal{E}^{n}\left(\mathbb{C}^{m}, g p\right)$ and $\mathcal{C}^{n}\left(\mathbb{C}^{m}, g p\right)$.

\subsection{Determinant cross ratios}

Here we construct certain non-constant holomorphic functions $\mathcal{E}^{n} \rightarrow \mathbb{C} \backslash\{0,1\}$.

Definition 2.8. Let $X$ be either $\mathbb{C P}^{m}$ or $\mathbb{C}^{m}$ and let $n \geq m+3$. For any $m+3$ dimensional multiindex $I=\left(i_{1}, \ldots, i_{m+3}\right)$ with distinct components $i_{t} \in\{1, \ldots, n\}$, set $\mathbf{i}=\left(i_{1}, \ldots, i_{m-1}\right), j=i_{m}, k=i_{m+1}, l=i_{m+2}, s=i_{m+3}$; the non-constant rational function

$$
e_{I}(q)=e_{\mathbf{i} ; j, k, l, s}(q)=\frac{d_{\mathbf{i}, j, k}(q)}{d_{\mathbf{i}, j, l}(q)}: \frac{d_{\mathbf{i}, k, s}(q)}{d_{\mathbf{i}, l, s}(q)}, \quad q \in\left(\mathbb{C P}^{m}\right)^{n},
$$


is called a determinant cross ratio, or, in brief, a DCR. This function is regular on the algebraic manifold $\mathcal{E}^{n}(X, g p) \subset\left(\mathbb{C P}^{m}\right)^{n}$.

The unordered set of indices $\{I\}=\left\{i_{1}, \ldots, i_{m-1}, j, k, l, s\right\}$ is called the support of the function $\mu=e_{I}=e_{\mathbf{i} ; j, k, l, s}$ and is denoted by supp $\mu$; its unordered subset $\{\mathbf{i}\}=$ $\left\{i_{1}, \ldots, i_{m-1}\right\}$ is called the essential support of $\mu$ and is denoted by $\operatorname{supp}_{\text {ess }} \mu$ (we often write I instead of $\{I\}$ and $\mathbf{i}$ instead of $\{\mathbf{i}\})$. In fact, $e_{I}(q)=e_{I}\left(q_{1}, \ldots, q_{n}\right)$ depends only on the vector variables $q_{t}$ with $t \in I$.

By a straightforward computation, one can easily obtain the following relations.

Lemma 2.9. (a) $e_{\mathbf{i} ;, t, r, s}=e_{\mathbf{i} ; j, k, r, s} / e_{\mathbf{i} ; j, k, r, t}$;

(b) $e_{\mathbf{j}, i ; j, k, r, s}=e_{\mathbf{j}, j ; i, k, r, s} e_{\mathbf{j}, s ; j, k, r, i}=e_{\mathbf{j}, k ; j, i, r, s} e_{\mathbf{j}, r ; j, k, i, s}$

The following lemma is a known fact of the classical invariant theory (for small dimensions it was discovered by A. F. Möbius [7], note especially Part 2). The proof may be extracted from [10, Section 2.14] (especially, Theorem 2.14.A).

Lemma 2.10. Let $\mathbf{i}=\left(i_{1}, \ldots, i_{m-1}\right)$ be a multiindex, and $j, k, l, s$ be indices such that all $i_{1}, \ldots, i_{m-1}, j, k, l$, s are distinct. Then $d_{\mathbf{i}, j, k} d_{\mathbf{i}, l, s}+d_{\mathbf{i}, j, l} d_{\mathbf{i}, s, k}+d_{\mathbf{i}, j, s} d_{\mathbf{i}, k, l}=0$.

Corollary 2.11. $e_{\mathbf{i} ; j, k, l, s}+e_{\mathbf{i} ; j, s, l, k}=1$.

Proof. By a straightforward computation and the above lemma, we see that

$$
e_{\mathbf{i} ; j, k, l, s}+e_{\mathbf{i} ; j, s, l, k}-1=\frac{d_{\mathbf{i}, j, k} d_{\mathbf{i}, l, s}+d_{\mathbf{i}, j, s} d_{\mathbf{i}, k, l}+d_{\mathbf{i}, j, l} d_{\mathbf{i}, s, k}}{d_{\mathbf{i}, j, l} d_{\mathbf{i}, k, s}}=0 .
$$

Lemma 2.12. Any $D C R$ omits the values 0 and 1 on $\mathcal{E}^{n}\left(\mathbb{C P}^{m}, g p\right)$.

Proof. Let $\lambda=e_{\mathbf{i}, j, k, l, s}$ be a DCR. By Definition $2.8, \lambda$ omits the value 0 . By Corollary $2.11 \lambda=1-e_{\mathbf{i} ; j, s, l, k}$. Since $e_{\mathbf{i} ; j, s, l, k}$ omits the value 0 , it follows that $\lambda$ does not take the value 1 either.

Remark 2.13. Notice that two determinant cross ratios, say $e_{I}=e_{\mathbf{i} ; j, k, l, s}$ and $e_{I^{\prime}}=$ $e_{\mathbf{i}^{\prime} ; j^{\prime}, k^{\prime}, l^{\prime}, s^{\prime}}$, coincide if and only if $\{\mathbf{i}\}=\left\{\mathbf{i}^{\prime}\right\}$ and $\left(j^{\prime}, k^{\prime}, l^{\prime}, s^{\prime}\right)$ is obtained from $(j, k, l, s)$ by a Kleinian permutation of four letters. The set of all determinant cross ratios is denoted by $\operatorname{DCR}\left(\mathcal{E}^{n}\right)=\operatorname{DCR}\left(\mathcal{E}^{n}(X, g p)\right)=\operatorname{DCR}\left(\mathcal{E}^{n}\left(\mathbb{C P}^{m}, g p\right)\right)$.

The $\mathbf{S}(n)$-action in $\mathcal{E}^{n}$ induces an $\mathbf{S}(n)$-action on functions defined by $(\sigma \lambda)(q)=$ $\lambda\left(\sigma^{-1} q\right)=\lambda\left(q_{\sigma(1)}, \ldots, q_{\sigma(n)}\right)\left(\lambda\right.$ is a function on $\mathcal{E}^{n}, q=\left(q_{1}, \ldots, q_{n}\right) \in \mathcal{E}^{n}$; notice that $\sigma \lambda$ may also be written as $\left(\sigma^{-1}\right)^{*} \lambda=\lambda \circ\left(\sigma^{-1}\right)$, where $\sigma$ and $\sigma^{-1}$ are considered as self-maps of $\mathcal{E}^{n}$ ). This action preserves the set of holomorphic functions.

Lemma 2.14. The $\mathbf{S}(n)$-action leaves the set $\mathrm{DCR}\left(\mathcal{E}^{n}\right)$ invariant. Moreover, this action is transitive on $\operatorname{DCR}\left(\mathcal{E}^{n}\right)$. 
Proof. Let $\mathbf{a}=\left(a_{1}, \ldots, a_{l}\right)$ be a multiindex. For any $\sigma \in \mathbf{S}(n)$, let $\sigma(\mathbf{a}):=$ $\left(\sigma\left(a_{1}\right), \ldots, \sigma\left(a_{l}\right)\right)$. Then $\sigma d_{\mathbf{a}}(q)=d_{\mathbf{a}}\left(\sigma^{-1} q\right)=d_{\sigma(\mathbf{a})}(q)$. Thus,

$$
\begin{aligned}
\sigma e_{\mathbf{i} ; j, k, r, s}(q) & =e_{\mathbf{i} ; j, k, r, s}\left(\sigma^{-1} q\right)=\frac{d_{\mathbf{i}, j, k}\left(\sigma^{-1} q\right)}{d_{\mathbf{i}, j, r}\left(\sigma^{-1} q\right)}: \frac{d_{\mathbf{i}, k, s}\left(\sigma^{-1} q\right)}{d_{\mathbf{i}, r, s}\left(\sigma^{-1} q\right)} \\
& =\frac{d_{\sigma(\mathbf{i}), \sigma(j), \sigma(k)}(q)}{d_{\sigma(\mathbf{i}), \sigma(j), \sigma(r)}(q)}: \frac{d_{\sigma(\mathbf{i}), \sigma(k), \sigma(s)}(q)}{d_{\sigma(\mathbf{i}), \sigma(r), \sigma(s)}(q)}=e_{\sigma(\mathbf{i}) ; \sigma(j), \sigma(k), \sigma(r), \sigma(s)}(q) .
\end{aligned}
$$

Let $e_{I}, e_{I^{\prime}} \in \operatorname{DCR}\left(\mathcal{E}^{n}\right)$. Since each of the sets $I$ and $I^{\prime}$ consists of $m+3 \leq n$ distinct elements of $\{1, \ldots, n\}$, there is $\sigma \in \mathbf{S}(n)$ such that $\sigma I=I^{\prime}$; hence $\sigma e_{I}=e_{I^{\prime}}$.

Lemma 2.15. DCRs are invariants of the PSL $(m+1)$-action on $\mathcal{E}^{n}\left(\mathbb{C P}^{m}, g p\right)$.

Proof. Clearly, the following elementary operators do not change a DCR:

$$
\begin{aligned}
& {\left[z_{1}: \cdots: z_{m+1}\right] \mapsto\left[a_{1} z_{1}: \cdots: a_{m+1} z_{m+1}\right] \quad \text { for } a_{1} \cdots: a_{m+1} \neq 0 ;} \\
& {\left[z_{1}: \cdots: z_{i}: \cdots: z_{j}: \cdots: z_{m+1}\right] \mapsto\left[z_{1}: \cdots: z_{j}: \cdots: z_{i}: \cdots: z_{m+1}\right]} \\
& {\left[z_{1}: \cdots: z_{i}: \cdots: z_{j}: \cdots: z_{m+1}\right] \mapsto\left[z_{1}: \cdots: z_{i}+z_{j}: \cdots: z_{j}: \cdots: z_{m+1}\right] .}
\end{aligned}
$$

Any element of PSL $(m+1)$ can be decomposed into a sequence of elementary operators. This proves the lemma.

Notation 2.16. For $s \in\{1, \ldots, m\}$, set $\mathbf{m}(\hat{s})=(1, \ldots, \hat{s}, \ldots, m)$. For $s=m$, we write sometimes $\widehat{\mathbf{m}}$ instead of $\mathbf{m}(\hat{m})$.

Lemma 2.17. (a) The map $P: M_{m, n} \rightarrow\left(\mathbb{C}^{n-m-2}\right)^{m}$ defined by

$$
q \mapsto P(q)=\left(\begin{array}{ccc}
p_{1, m+3}(q), & \ldots, & p_{1, n}(q) \\
\ldots & \ldots & \ldots \\
p_{m, m+3}(q), & \ldots & p_{m, n}(q)
\end{array}\right),
$$

with $p_{s, t}(q)=e_{\mathbf{m}(\hat{s}) ; s, m+1, m+2, t}(q)$ for $s=1, \ldots, m$ and $t=m+3, \ldots, n$, is $a$ holomorphic embedding.

(b) $M_{m, n}$ is a hyperbolic space.

Proof. (a) For $q=\left(q_{1}, \ldots, q_{n}\right) \in M_{m, n}$ with $q_{i}=\left[\begin{array}{ll}z_{i, 1} & : \cdots: z_{i, m+1}\end{array}\right]$ we have $z_{t, m+1}=d_{\widehat{\mathbf{m}}, m, t}(q) \neq 0$ and $z_{t, s}= \pm d_{\mathbf{m}(\hat{s}), m+1, t}(q) \neq 0$. Furthermore, $d_{\mathbf{m}(\hat{s}), s, m+1}(q)=d_{\mathbf{m}(\hat{s}), s, m+2}(q)=(-1)^{m-s}, d_{\mathbf{m}(\hat{s}), m+1, t}(q)=-(-1)^{m-s} z_{t, s}$ and $d_{\mathbf{m}(\hat{s}), m+2, t}(q)=(-1)^{m-s}\left(z_{t, m+1}-z_{t, s}\right) ;$ thus,

$$
p_{s, t}(q)=\frac{d_{\mathbf{m}(\hat{s}), s, m+1}(q)}{d_{\mathbf{m}(\hat{s}), s, m+2}(q)}: \frac{d_{\mathbf{m}(\hat{s}), m+1, t}(q)}{d_{\mathbf{m}(\hat{s}), m+2, t}(q)}=1-\frac{z_{t, m+1}}{z_{t, s}} .
$$

If $q^{\prime}=\left(q_{1}^{\prime}, \ldots, q_{n}^{\prime}\right) \in M_{m, n}$ with $q_{i}^{\prime}=\left[z_{i, 1}^{\prime}: \cdots: z_{i, m+1}^{\prime}\right]$ and $p_{s, t}(q)=p_{s, t}\left(q^{\prime}\right)$ for $s=1, \ldots, m$, then

$$
\frac{z_{t, m+1}}{z_{t, s}}=\frac{z_{t, m+1}^{\prime}}{z_{t, s}^{\prime}} \quad \text { for all } s=1, \ldots, m
$$


and hence $q_{t}=q_{t}^{\prime}$. Thus, $P(q)=P\left(q^{\prime}\right)$ implies $q=q^{\prime}$ and so $P$ is injective. To see that $P$ is an embedding, it suffices to observe that from the above calculation it follows that at any point the Jacobi matrix of $P$ is of maximal rank.

(b) Since every DCR omits the values 0 and $1, P\left(M_{m, n}\right) \subset(\mathbb{C} \backslash\{0,1\})^{m(n-m-2)}$. Hence $M_{m, n}$ is hyperbolic.

\section{Holomorphic functions $\mathcal{E}^{n} \rightarrow \mathbb{C} \backslash\{0,1\}$}

Notation 3.1. For a complex space $Z$, we denote by $L(Z)$ the set of all non-constant holomorphic functions $Z \rightarrow \mathbb{C} \backslash\{0,1\}$.

The following lemma plays a crucial part in the explicit description of $L\left(\mathcal{E}^{n}\right)$.

Lemma 3.2. Let $A, B, C \in \mathbb{C}\left[\mathbb{C}^{m n}\right]=\mathbb{C}\left[z_{1,1}, \ldots, z_{n, m}\right]$ be pairwise co-prime polynomials on $\mathbb{C}^{m n}$ non-vanishing on $\mathcal{E}^{n}\left(\mathbb{C}^{m}\right.$, gp $) \subset \mathbb{C}^{m n}$. Assume that at least one of them is non-constant and $A+B+C=0$. Then there exist a multiindex $\mathbf{i}=\left(i_{1}, \ldots, i_{m-1}\right)$, indices $j, k, l, s$, and $\alpha \in \mathbb{C} \backslash\{0\}$ such that all $i_{1}, \ldots, i_{m-1}, j, k, l, s$ are distinct and $A=\alpha d_{\mathbf{i}, j, k} d_{\mathbf{i}, l, s}, B=\alpha d_{\mathbf{i}, j, l} d_{\mathbf{i}, s, k}$ and $C=\alpha d_{\mathbf{i}, j, s} d_{\mathbf{i}, k, l}$.

Proof. Since $A, B, C$ do not vanish on $\mathcal{E}^{n}\left(\mathbb{C}^{m}, g p\right)=\mathbb{C}^{m n} \backslash \bigcup_{\mathbf{i}}\left\{q \in \mathbb{C}^{m n} \mid d_{\mathbf{i}}(q)=0\right\}$, from Lemma 2.1 we see that

$$
A=\alpha \prod_{\mathbf{i} \in \mathbf{I}^{m+1}} d_{\mathbf{i}}^{a_{\mathbf{i}}}, \quad B=\beta \prod_{\mathbf{i} \in \mathbf{I}^{m+1}} d_{\mathbf{i}}^{b_{\mathbf{i}}}, \quad C=\gamma \prod_{\mathbf{i} \in \mathbf{I}^{m+1}} d_{\mathbf{i}}^{c_{\mathbf{i}}},
$$

where $\alpha, \beta, \gamma \in \mathbb{C} \backslash\{0\}$ and $a_{\mathbf{i}}, b_{\mathbf{i}}, c_{\mathbf{i}} \in \mathbb{Z}_{+}$(see Notation 2.2). The polynomials are homogeneous; thus, the equality $A+B+C=0$ implies that $\operatorname{deg} A=\operatorname{deg} B=\operatorname{deg} C$, i.e. $\sum a_{\mathbf{i}}=\sum b_{\mathbf{i}}=\sum c_{\mathbf{i}}$. For every index $i_{0}, 1 \leq i_{0} \leq n$, we can write

$$
A=A_{i_{0}} \prod_{\mathbf{i} \in \mathbf{I}_{i_{0}}^{m}} d_{i_{0}, \mathbf{i}}^{a_{i_{0}, \mathbf{i}},} \quad B=B_{i_{0}} \prod_{\mathbf{i} \in \mathbf{I}_{i_{0}}^{m}} d_{i_{0}, \mathbf{i}}^{b_{i_{0}, \mathbf{i}}}, \quad C=C_{i_{0}} \prod_{\mathbf{i} \in \mathbf{I}_{i_{0}}^{m}} d_{i_{0}, \mathbf{i}}^{c_{i_{0}}, \mathbf{i}},
$$

where $A_{i_{0}}, B_{i_{0}}, C_{i_{0}}$ are the products of all factors $d_{\mathbf{i}}$ that do not contain the variables $z_{i_{0}, 1}, \ldots, z_{i_{0}, m}$, i.e.

$$
A_{i_{0}}= \pm \alpha \prod_{\mathbf{i} \in \mathbf{I}_{i_{0}}^{m+1}} d_{\mathbf{i}}^{a_{\mathbf{i}}}, \quad B_{i_{0}}= \pm \beta \prod_{\mathbf{i} \in \mathbf{I}_{i_{0}}^{m+1}} d_{\mathbf{i}}^{b_{\mathbf{i}}}, \quad C_{i_{0}}= \pm \gamma \prod_{\mathbf{i} \in \mathbf{I}_{i_{0}}^{m+1}} d_{\mathbf{i}}^{c_{\mathbf{i}}}
$$

The proof is divided into two steps.

Step 1. Let us prove the following statement:

(*) There is an index $t_{0}$ such that the polynomials $A_{t_{0}}, B_{t_{0}}, C_{t_{0}}$ are constant. 
Proof. First, we prove that for any $i_{0} \in\{1, \ldots, n\}$,

$$
\sum a_{i_{0}, \mathbf{i}}=\sum b_{i_{0}, \mathbf{i}}=\sum c_{i_{0}, \mathbf{i}} \quad \text { and } \quad \operatorname{deg} A_{i_{0}}=\operatorname{deg} B_{i_{0}}=\operatorname{deg} C_{i_{0}} .
$$

Without loss of generality, we can assume that either (a) $\sum a_{i_{0}, \mathbf{i}}>\sum b_{i_{0}, \mathbf{i}} \geq \sum c_{i_{0}, \mathbf{i}}$ or (b) $\sum a_{i_{0}, \mathbf{i}}=\sum b_{i_{0}, \mathbf{i}}>\sum c_{i_{0}, \mathbf{i}}$ or (c) $\sum a_{i_{0}, \mathbf{i}}=\sum b_{i_{0}, \mathbf{i}}=\sum c_{i_{0}, \mathbf{i}}$.

Compare the terms of the maximal degree in the variable $z_{i_{0}, 1}$ in the equality $A+B+$ $C=0$. In case (a) we have $A_{i_{0}} \prod \delta_{\mathbf{i} ; 1}^{a_{i}, \mathbf{i}}=0$ (see Notation 2.2 for the definition of $\delta_{\mathbf{i} ; k}$ ). This means $A=0$, a contradiction. In case (b) we obtain

$$
A_{i_{0}} \prod_{\mathbf{i} \in \mathbf{I}_{i_{0}}^{m}} \delta_{\mathbf{i}}^{a_{i, 1}, \mathbf{i}}+B_{i_{0}} \prod_{\mathbf{i} \in \mathbb{I}_{i_{0}}^{m}} \delta_{\mathbf{i}_{i}, 1}^{b_{i, \mathbf{i}}}=0 .
$$

By Lemma 2.1. we see that the greatest common divisors $\operatorname{GCD}\left(A_{i_{0}}, \prod_{\mathbf{i} \in \mathbf{I}_{i_{0}}^{m}} \delta_{\mathbf{i} ; 1}^{b_{i_{0}} \mathbf{i}}\right)$ and $\operatorname{GCD}\left(B_{i_{0}}, \prod_{\mathbf{i} \in \mathbf{I}_{i_{0}}^{m}} \delta_{\mathbf{i} ; 1}^{a_{i_{0}, \mathbf{i}}}\right)$ equal 1 ; hence $A_{i_{0}}+B_{i_{0}}=0$ and $a_{i_{0}, \mathbf{i}}=b_{i_{0}, \mathbf{i}}$ for all $\mathbf{i} \in \mathbf{I}_{i_{0}}^{m}$. Thus $C=-(A+B) \stackrel{=}{=}$, a contradiction. This completes the proof of 44 . Since $A+B+C=0$, for each $k=1, \ldots, m$ the leading term of $A+B+C$ in the variable $z_{i_{0}, k}$ is 0 , i.e.

$$
A_{i_{0}} \prod_{\mathbf{i} \in \mathbf{I}_{i_{0}}^{m}} \delta_{\mathbf{i} ; k}^{a_{i_{0}, \mathbf{i}}}+B_{i_{0}} \prod_{\mathbf{i} \in \mathbf{I}_{i_{0}}^{m}} \delta_{\mathbf{i} ; k}^{b_{i_{0}, \mathbf{i}}}+C_{i_{0}} \prod_{\mathbf{i} \in I_{i_{0}}^{m}} \delta_{\mathbf{i} ; k k}^{c_{i_{0}, \mathbf{i}}}=0 .
$$

Assume that there is $i_{0}$ such that $A_{i_{0}} \neq A$ and $A_{i_{0}} \neq$ const (otherwise either $A=$ const, which is a contradiction, or $A_{i_{0}}=$ const and $(*)$ holds with $t_{0}=i_{0}$ ). By (4), for such $i_{0}$ we have $0<\operatorname{deg} A_{i_{0}}=\operatorname{deg} B_{i_{0}}=\operatorname{deg} C_{i_{0}}<\operatorname{deg} A=\operatorname{deg} B=\operatorname{deg} C$. By Lemma 2.1. all $\delta_{\mathbf{i} ; k}$ are irreducible and distinct. Since $A, B, C$ are pairwise co-prime, the integers $a_{i_{0}, \mathbf{i}}, b_{i_{0}, \mathbf{i}}, c_{i_{0}, \mathbf{i}}$ are also distinct. Using these facts, it is easy to verify that the second order system of the linear equations in variables $A_{i_{0}}, B_{i_{0}}, C_{i_{0}}$ defined by (5) with $k=1,2$ is of rank 2; actually, all its $2 \times 2$ minors are non-zero polynomials. Thus, $A_{i_{0}} / \mathfrak{A}=B_{i_{0}} / \mathfrak{B}=$ $C_{i_{0}} / \mathfrak{C}$ with certain non-zero polynomials $\mathfrak{A}, \mathfrak{B}, \mathfrak{C}$. The polynomials $B_{i_{0}}$ and $C_{i_{0}}$ are coprime, so

$$
\mathfrak{B}=\tilde{B} \cdot B_{i_{0}} \quad \text { and } \quad \mathfrak{C}=\tilde{C} \cdot C_{i_{0}},
$$

where $\tilde{B}, \tilde{C}$ are non-zero polynomials. Pick $\mathbf{i}_{0}=\left(i_{1}, \ldots, i_{m}\right) \in \mathbf{I}_{i_{0}}^{m}$ such that $a_{i_{0}, \mathbf{i}_{0}}>0$. Since $A, B$ and $C$ are pairwise co-prime, it follows that $b_{i_{0}, \mathrm{i}_{0}}=c_{i_{0}, \mathrm{i}_{0}}=0$.

Let us prove that

$$
A=A_{i_{0}} d_{i_{0}, \mathbf{i}_{0}}^{a_{i_{0}, \mathbf{i}_{0}}}, \quad B_{i_{0}}= \pm \beta \prod_{i \neq i_{0}} d_{i, \mathbf{i}_{0}}^{b_{i, \mathbf{i}_{0}}}, \quad C_{i_{0}}= \pm \gamma \prod_{i \neq i_{0}} d_{i, \mathbf{i}_{0}}^{c_{i, \mathbf{i}_{0}}}
$$

the statement $(*)$ will follow from this by combinatorial considerations.

Let $L$ be the linear subspace of $\mathbb{C}^{m n}$ defined by $z_{i_{1}, 2}=\cdots=z_{i_{m}, 2}$. Since for $\mathbf{i} \in \mathbf{I}_{i_{0}}^{m}$ we have $\left.\delta_{\mathbf{i} ; k}\right|_{L}=0$ if and only if $\#\left(\mathbf{i} \cap \mathbf{i}_{0}\right)=m$ and $k \neq 2$, the restrictions

$$
\left.\mathfrak{B}\right|_{L}=-\left.\left(\prod \delta_{\mathbf{i} ; 2}^{a_{0}, \mathbf{i}} \cdot \prod \delta_{\mathbf{i} ; 1}^{c_{i_{0}, \mathbf{i}}}\right)\right|_{L} \quad \text { and }\left.\quad \mathfrak{C}\right|_{L}=-\left.\left(\prod \delta_{\mathbf{i} ; 2}^{a_{i}, \mathbf{i}} \cdot \prod \delta_{\mathbf{i} ; 1}^{b_{i}, \mathbf{i}}\right)\right|_{L}
$$


are non-zero polynomials. Thus $\left.\tilde{B}\right|_{L},\left.\tilde{C}\right|_{L} \neq 0$. According to $\left[8,\left.\mathfrak{B}\right|_{L}\right.$ and $\left.\mathfrak{C}\right|_{L}$ are products of irreducible polynomials of degree $\leq m-1$. By (3),

$$
\left.B_{i_{0}}\right|_{L}= \pm \beta \prod_{\mathbf{i} \in \mathbf{I}_{i_{0}}^{m+1}}\left(\left.d_{\mathbf{i}}\right|_{L}\right)^{b_{\mathbf{i}}} \quad \text { and }\left.\quad C_{i_{0}}\right|_{L}= \pm \gamma \prod_{\mathbf{i} \in \mathbf{I}_{i_{0}}^{m+1}}\left(\left.d_{\mathbf{i}}\right|_{L}\right)^{c_{\mathbf{i}}}
$$

By $(6),\left.B_{i_{0}}\right|_{L}$ and $\left.C_{i_{0}}\right|_{L}$ must also be products of irreducible polynomials of degree $\leq$ $m-1$. By Lemma 2.3 this may happen if and only if the decompositions 90 of $\left.B_{i_{0}}\right|_{L}$ and $\left.C_{i_{0}}\right|_{L}$ contain only factors of the form $\left.d_{i, \mathbf{i}_{0}}\right|_{L}$, i.e. we have shown that

$$
B_{i_{0}}= \pm \beta \prod_{i \neq i_{0}} d_{i, \mathbf{i}_{0}}^{b_{i, \mathbf{i}_{0}}} \quad \text { and } \quad C_{i_{0}}= \pm \gamma \prod_{i \neq i_{0}} d_{i, \mathbf{i}_{0}}^{c_{i, \mathbf{i}_{0}}}
$$

To complete the proof of (7), we must show that $a_{i_{0}, \mathbf{i}_{1}}=0$ for any $\mathbf{i}_{1} \in \mathbf{I}_{i_{0}}^{m} \backslash\left\{\mathbf{i}_{0}\right\}$. Suppose the contrary, that there is $\mathbf{i}_{1} \in \mathbf{I}_{i_{0}}^{m} \backslash\left\{\mathbf{i}_{0}\right\}$ such that $a_{i_{0}, \mathbf{i}_{1}}>0$. Then similarly we have

$$
B_{i_{0}}= \pm \beta \prod_{i \neq i_{0}} d_{i, \mathbf{i}_{0}}^{b_{i, \mathbf{i}_{0}}}= \pm \beta^{\prime} \prod_{i \neq i_{0}} d_{i, \mathbf{i}_{1}}^{b_{i, \mathbf{i}_{1}}}, \quad C_{i_{0}}= \pm \gamma \prod_{i \neq i_{0}} d_{i, \mathbf{i}_{0}}^{c_{i, \mathbf{i}_{0}}}= \pm \gamma^{\prime} \prod_{i \neq i_{0}} d_{i, \mathbf{i}_{1}}^{c_{i, \mathbf{i}_{1}}}
$$

Since we assume that $B_{i_{0}}, C_{i_{0}} \neq$ const, 10 can occur only if $B_{i_{0}}= \pm \beta d_{s, \mathbf{i}_{0}}^{b_{s, \mathbf{i}_{0}}}$ and $C_{i_{0}}= \pm \gamma d_{s, \mathbf{i}_{0}}^{c_{s, \mathbf{i}_{0}}}$, i.e. $B_{i_{0}}$ and $C_{i_{0}}$ have a non-trivial common factor, which contradicts our assumptions. Thus, we have proved (7).

By (4), the choice of $i_{0}$ implies that $\sum a_{i_{0}, \mathbf{i}}=\sum b_{i_{0}, \mathbf{i}}=\sum c_{i_{0}, \mathbf{i}}>0$. Since $b_{i_{0}, \mathbf{i}_{0}}=0$, the latter implies that there exists a multiindex $\mathbf{i}_{2} \neq \mathbf{i}_{0}$ such that $b_{i_{0}, \mathbf{i}_{2}}>0$. In the same way as we proved (7), we conclude that

$$
A_{i_{0}}= \pm \alpha^{\prime} \prod_{i \neq i_{0}} d_{i, \mathbf{i}_{2}}^{a_{i, \mathbf{i}_{2}}}, \quad B=B_{i_{0}} d_{i_{0}, \mathbf{i}_{2}}^{b_{i_{0}, \mathbf{i}_{2}}}, \quad C_{i_{0}}= \pm \gamma^{\prime} \prod_{i \neq i_{0}} d_{i, \mathbf{i}_{2}}^{c_{i, \mathbf{i}_{2}}} .
$$

Comparing (7) and (11), we conclude that $C_{i_{0}}= \pm \gamma d_{s, \mathbf{i}_{0}}^{c_{s, \mathbf{i}_{0}}}$ and $\mathbf{i}_{0} \cap \mathbf{i}_{2} \neq \emptyset$. Pick $t_{0} \in \mathbf{i}_{0} \cap \mathbf{i}_{2}$. Then $A_{t_{0}}=$ const. Due to (4), $\operatorname{deg} A_{i_{0}}=\operatorname{deg} B_{i_{0}}=\operatorname{deg} C_{i_{0}}$; that is, $A_{t_{0}}, B_{t_{0}}, C_{t_{0}}$ are constant polynomials. This completes the proof of $(*)$.

Step 2. Let $i_{0} \in\{1, \ldots, n\}$. Let us prove the following statement:

(**) Assume that $A_{i_{0}}, B_{i_{0}}, C_{i_{0}}$ are constant. Then there are $\mathbf{i}=\left(i_{1}, \ldots, i_{m-2}\right)$, indices $j, s, l, t$, and $\alpha \in \mathbb{C} \backslash\{0\}$ such that all $i_{0}, i_{1}, \ldots, i_{m-2}, j, s, l, t$ are distinct and $A=\alpha d_{i_{0}, \mathbf{i}, j, s} d_{i_{0}, \mathbf{i}, l, t}, B=\alpha d_{i_{0}, \mathbf{i}, j, l} d_{i_{0}, \mathbf{i}, t, s}$ and $C=\alpha d_{i_{0}, \mathbf{i}, j, t} d_{i_{0}, \mathbf{i}, s, l}$.

Proof. Set $A^{\prime}=A_{i_{0}} \prod \delta_{\mathbf{i} ; 1}^{a_{i_{0}, \mathbf{i}}}, B^{\prime}=B_{i_{0}} \prod \delta_{\mathbf{i} ; 1}^{b_{i_{0}, \mathbf{i}}}, C^{\prime}=C_{i_{0}} \prod \delta_{\mathbf{i} ; 1}^{c_{i_{0}, \mathbf{i}}}$. It is easily seen that $A^{\prime}$, $B^{\prime}$ and $C^{\prime}$ are pairwise co-prime on $\mathbb{C}^{(m-1)(n-1)}$ and do not vanish on the configuration space $\mathcal{E}^{n-1}\left(\mathbb{C}^{m-1}, g p\right) \subset \mathbb{C}^{(m-1)(n-1)}$. According to [5), $A^{\prime}+B^{\prime}+C^{\prime}=0$. The proof of $(* *)$ is by induction on $m$.

Let $m=2$. Lemma 5.1 of [6] states that for any three non-constant pairwise co-prime polynomials $P=a \prod_{i \neq j}\left(x_{i}-x_{j}\right)^{a_{i, j}}, Q=b \prod_{i \neq j}\left(x_{i}-x_{j}\right)^{b_{i, j}}, R=c \prod_{i \neq j}\left(x_{i}-x_{j}\right)^{c_{i, j}}$ 
in the variables $x_{1}, \ldots, x_{n}$ which satisfy the equation $P+Q+R=0$, there are distinct indices $j, s, l, t$ and $\alpha \in \mathbb{C} \backslash\{0\}$ such that either $P=\alpha\left(x_{j}-x_{s}\right), Q=\alpha\left(x_{s}-x_{l}\right), R=$ $\alpha\left(x_{l}-x_{j}\right)$ or $P=\alpha\left(x_{j}-x_{s}\right)\left(x_{l}-x_{t}\right), Q=\alpha\left(x_{j}-x_{l}\right)\left(x_{t}-x_{s}\right), R=\alpha\left(x_{j}-x_{t}\right)\left(x_{s}-x_{l}\right)$. This lemma applies to the polynomials $A^{\prime}, B^{\prime}, C^{\prime}$ in the variables $z_{1,2}, \ldots, z_{n, 2}$. Therefore, there are distinct indices $j, s, l, t$ and $\alpha \in \mathbb{C} \backslash\{0\}$ such that either $A^{\prime}=\alpha\left(z_{j, 2}-z_{s, 2}\right)$, $B^{\prime}=\alpha\left(z_{s, 2}-z_{l, 2}\right), C^{\prime}=\alpha\left(z_{l, 2}-z_{j, 2}\right)$ or $A^{\prime}=\alpha\left(z_{j, 2}-z_{s, 2}\right)\left(z_{l, 2}-z_{t, 2}\right), B^{\prime}=$ $\alpha\left(z_{j, 2}-z_{l, 2}\right)\left(z_{t, 2}-z_{s, 2}\right), C^{\prime}=\alpha\left(z_{j, 2}-z_{t, 2}\right)\left(z_{s, 2}-z_{l, 2}\right)$. Thus, for our original polynomials $A, B, C$ we infer that either $A=\alpha d_{i_{0}, j, s}, B=\alpha d_{i_{0}, s, l}, C=\alpha d_{i_{0}, l, j}$ or $A=\alpha d_{i_{0}, j, s} d_{i_{0}, l, t}, B=\alpha d_{i_{0}, j, l} d_{i_{0}, t, s}, C=\alpha d_{i_{0}, j, t} d_{i_{0}, s, l}$. It is easily seen that in the first case $A+B+C=\alpha\left(d_{i_{0}, j, s}+d_{i_{0}, s, l}+d_{i_{0}, l, j}\right) \not \equiv 0$. Thus, the equality $A+B+C=0$ can hold only in the second case, which provides the base of induction.

Suppose that (**) is satisfied for some $m=k-1>1$, and let us prove it for $m=k$. Due to (*) (from Step 1), the induction hypothesis applies to the polynomials $A^{\prime}, B^{\prime}, C^{\prime}$; that is, there exist a multiindex $\mathbf{i}=\left(i_{1}, \ldots, i_{k-2}\right)$, indices $j, s, l, t$, and $\alpha \in \mathbb{C} \backslash\{0\}$ such that all $i_{1}, \ldots, i_{k-2}, j, s, l, t$ are distinct and $A^{\prime}=\alpha \cdot d_{\mathbf{i}, j, s} \cdot d_{\mathbf{i}, l, t}, B^{\prime}=\alpha \cdot d_{\mathbf{i}, j, l} \cdot d_{\mathbf{i}, t, s}$ and $C^{\prime}=\alpha \cdot d_{\mathbf{i}, j, t} \cdot d_{\mathbf{i}, s, l}$; i.e. $A, B$ and $C$ can be written as $A=\alpha \cdot d_{i_{0}, \mathbf{i}, j, s} \cdot d_{i_{0}, \mathbf{i}, l, t}$, $B=\alpha \cdot d_{i_{0}, \mathbf{i}, j, l} \cdot d_{i_{0}, \mathbf{i}, t, s}$ and $C=\alpha \cdot d_{i_{0}, \mathbf{i}, j, t} \cdot d_{i_{0}, \mathbf{i}, s, l}$. This completes the proofs of $(* *)$ and of Lemma 3.2 .

Theorem 3.3. Let $X=\mathbb{C}^{m}$ or $\mathbb{C P}^{m}$. Then $L\left(\mathcal{E}^{n}(X, g p)\right)=\operatorname{DCR}\left(\mathcal{E}^{n}(X, g p)\right)$.

Proof. By Corollary 2.12 it suffices to show that $L\left(\mathcal{E}^{n}(X, g p)\right) \subseteq \operatorname{DCR}\left(\mathcal{E}^{n}(X, g p)\right)$. We follow [6]. Let $\mu \in L\left(\mathcal{E}^{n}(X, g p)\right)$, that is, $\mu: \mathcal{E}^{n}(X, g p) \rightarrow \mathbb{C} \backslash\{0,1\}$ is a holomorphic function. First, let $X=\mathbb{C}^{m}$. It follows from the Big Picard Theorem that $\mu$ is a regular function on $\mathcal{E}^{n}\left(\mathbb{C}^{m}, g p\right)$; hence it is a rational function on $\left(\mathbb{C}^{m}\right)^{n}$ and there are co-prime polynomials $A, B \in \mathbb{C}\left[\left(\mathbb{C}^{m}\right)^{n}\right]$ that do not vanish on $\mathcal{E}^{n}\left(\mathbb{C}^{m}, g p\right)$ such that $\mu=-A / B$. The function $1-\mu=(A+B) / B$ also omits the values 0,1 . The polynomials $A, B$ and $C=-B-A$ are pairwise co-prime, do not vanish on $\mathcal{E}^{n}\left(\mathbb{C}^{m}, g p\right)$ and satisfy $A+B+$ $C=0$. Lemma 3.2 applies to the last three polynomials and shows that $\mu=-A / B=$ $-d_{\mathbf{i}, j, k} d_{\mathbf{i}, l, s} / d_{\mathbf{i}, j, l} d_{\mathbf{i}, s, k}=e_{\mathbf{i} ; j, k, l, s}$ for appropriate $\mathbf{i}, j, k, l, s$. When $X=\mathbb{C P}^{m}$, we restrict $\mu$ from $\mathcal{E}^{n}\left(\mathbb{C P}^{m}, g p\right)$ to $\mathcal{E}^{n}\left(\mathbb{C}^{m}, g p\right)$ and apply the above result, which leads to the desired conclusion.

\section{Simplicial structure on $L\left(\mathcal{E}^{n}\right)$}

It was shown in [6] that the set $L(Z)$ of all non-constant holomorphic functions $Z \rightarrow$ $\mathbb{C} \backslash\{0,1\}$ on a complex space $Z$ may be endowed with a natural structure of a simplicial complex $L_{\Delta}(Z)$, and the correspondence $Z \mapsto L_{\Delta}(Z)$ has some properties of a contravariant functor from the category of complex spaces to the category of simplicial complexes. First, we recall the definition of the complex $L_{\Delta}(Z)$.

Definition 4.1. Let $Z$ be a complex space and $L(Z)$ be the set of all non-constant holomorphic functions $Z \rightarrow \mathbb{C} \backslash\{0,1\}$. For $\mu, v \in L(Z)$, we say that $v$ is a proper divisor of $\mu$ and write $\nu \mid \mu$ if $\mu: v \in L(Z)$. Clearly, $\nu \mid \mu$ is equivalent to $\mu \mid \nu$. 
A non-empty ordered subset $\Delta=\left\{\mu_{0}, \ldots, \mu_{s}\right\} \subseteq L(Z)$ is said to be an s-simplex with vertices $\mu_{0}, \ldots, \mu_{s}$ if $\mu_{i} \mid \mu_{j}$ for all $i \neq j$. Since any non-empty subset of a simplex is also a simplex, we obtain a well-defined simplicial complex $L_{\Delta}(Z)$ with the set of vertices $L(Z)$. If $Z$ is a quasi-projective algebraic variety, then the set $L(Z)$ consists of a finite number of regular functions and the complex $L_{\Delta}(Z)$ is finite (it can be empty). A holomorphic map $f: Z \rightarrow Y$ of complex spaces induces the homomorphism $f^{*}: \mathcal{O}(Y) \rightarrow \mathcal{O}(Z)$ of the algebras of holomorphic functions defined, as usual, by $f^{*}(\lambda)=\lambda \circ f, \lambda \in \mathcal{O}(Y)$. Let $\lambda \in L(Y)$; suppose that $f^{*}(\lambda) \neq$ const. Then the map of the vertices $f^{*}: L(Y) \ni \lambda \mapsto \lambda \circ f \in L(Z)$ induces the simplicial map $f^{*}: L_{\Delta}(Y) \rightarrow L_{\Delta}(Z)$ whose restriction to each simplex $\Delta \subseteq L(Y)$ is injective and preserves dimensions of simplices.

Remark 4.2. Let $\mathbf{i}=\left(i_{1}, \ldots, i_{m-1}\right)$ and $\mu=e_{\mathbf{i} ; j, k, s, t}$. Let $\mathbf{e}_{1}, \ldots, \mathbf{e}_{m}$ be the standard basis in $\mathbb{C}^{m}, \mathbf{u}_{1}=0$ and $\mathbf{u}_{p}=\sum_{j=m-p+2}^{m} \mathbf{e}_{j}$ for $p=2, \ldots, m-1$. Set

$$
L=\left\{\left(v_{1}, \ldots, v_{n}\right) \in\left(\mathbb{C}^{m}\right)^{n} \mid v_{i_{p}}=\mathbf{u}_{p} \text { for } p=1, \ldots, m-1\right\} .
$$

For $q=\left(q_{1}, \ldots, q_{n}\right) \in\left(\mathbb{C}^{m}\right)^{n} \cap L$ with all $q_{r}=\left(z_{r, 1}, \ldots, z_{r, m}\right) \in \mathbb{C}^{m}$, the restriction of any $d_{\mathbf{i}, l, r}$ to the subspace $L$ reduces to a certain determinant of order 2 and may be computed as $(-1)^{m}\left(z_{l, 1} z_{r, 2}-z_{r, 1} z_{l, 2}\right)$. Consequently, the restriction of $\mu$ to $L$ may be written as

$$
\left.\mu\right|_{L}(q)=\frac{z_{j, 1} / z_{j, 2}-z_{k, 1} / z_{k, 2}}{z_{j, 1} / z_{j, 2}-z_{s, 1} / z_{s, 2}}: \frac{z_{k, 1} / z_{k, 2}-z_{t, 1} / z_{t, 2}}{z_{s, 1} / z_{s, 2}-z_{t, 1} / z_{t, 2}} .
$$

Thus, $\left.\mu\right|_{L}$ is the cross ratio of the four quantities $a=z_{j, 1} / z_{j, 2}, b=z_{k, 1} / z_{k, 2}, c=$ $z_{j, 1} / z_{j, 2}$ and $d=z_{t, 1} / z_{t, 2}$, which may be treated as four distinct points in $\mathbb{C P}^{1}$ whenever $q \in \mathcal{E}^{n} \cap L$. If $\mu$ and $\mu^{\prime}$ are determinant cross ratios, $\mu \mid \mu^{\prime}$ and $\mathbf{i}=\operatorname{supp}_{\text {ess }} \mu=\operatorname{supp}_{\text {ess }} \mu^{\prime}$, then $\operatorname{supp}_{\mathrm{ess}}\left(\mu: \mu^{\prime}\right)=\operatorname{supp}_{\mathrm{ess}} \mu=\operatorname{supp}_{\mathrm{ess}} \mu^{\prime}$. In view of the above, the restrictions of $\mu, \mu^{\prime}$ and $\mu: \mu^{\prime}$ to $L$ are usual cross ratios, and moreover, $\left(\left.\mu\right|_{L}\right):\left(\left.\mu^{\prime}\right|_{L}\right)=\left.\left(\mu: \mu^{\prime}\right)\right|_{L}$.

In view of the previous remark, certain results about usual cross ratios also apply to the DCRs. In particular, we shall use the following lemma (see Lemma 5.7 of [6]):

Lemma 4.3. If the ratio $\mu: \mu^{\prime}$ of two cross ratios

$$
\mu=\frac{q_{j}-q_{k}}{q_{j}-q_{s}}: \frac{q_{k}-q_{t}}{q_{s}-q_{t}} \quad \text { and } \quad \mu^{\prime}=\frac{q_{j^{\prime}}-q_{k^{\prime}}}{q_{j^{\prime}}-q_{s^{\prime}}}: \frac{q_{k^{\prime}}-q_{t^{\prime}}}{q_{s^{\prime}}-q_{t^{\prime}}}
$$

is a cross ratio of certain four of the eight variables $q_{j}, q_{k}, q_{s}, q_{t}, q_{j^{\prime}}, q_{k^{\prime}}, q_{s^{\prime}}, q_{t^{\prime}}$ then $\#\left(\{j, k, s, t\} \cap\left\{j^{\prime}, k^{\prime}, s^{\prime}, t^{\prime}\right\}\right)=3$ and $\mu^{\prime}$ is obtained from $\mu$ by replacing one of the variables $q_{j}, q_{k}, q_{s}, q_{t}$ with some $q_{m}$, where $m \neq j, k, s, t$.

We also need the following technical lemma.

Lemma 4.4. Let $X=\mathbb{C P}^{m}$ or $\mathbb{C}^{m}, n>m+2$, and let $\mu=e_{\mathbf{i} ; j, k, r, s}$ be a proper divisor of a determinant cross ratio $\mu^{\prime}$. Then $\#\left(\operatorname{supp}_{\mathrm{ess}} \mu \cap \operatorname{supp}_{\mathrm{ess}} \mu^{\prime}\right) \geq m-2$. If $\operatorname{supp} \mu^{\prime} \neq \operatorname{supp} \mu$,

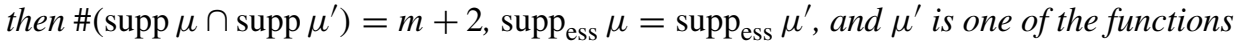
$e_{\mathbf{i} ; j, k, r, t}, e_{\mathbf{i} ; k, j, s, t}, e_{\mathbf{i} ; r, s, j, t}, e_{\mathbf{i} ; s, r, k, t}$. 
Proof. Set

$$
\mu=\frac{d_{\mathbf{i}, j, k}}{d_{\mathbf{i}, j, r}}: \frac{d_{\mathbf{i}, k, s}}{d_{\mathbf{i}, r, s}}, \quad \mu^{\prime}=\frac{d_{\mathbf{i}^{\prime}, j^{\prime}, k^{\prime}}}{d_{\mathbf{i}^{\prime}, j^{\prime}, r^{\prime}}}: \frac{d_{\mathbf{i}^{\prime}, k^{\prime}, s^{\prime}}}{d_{\mathbf{i}^{\prime}, r^{\prime}, s^{\prime}}} .
$$

Let $\mathbf{j}=\mathbf{i} \cap \mathbf{i}^{\prime}$. Since $\mu \mid \mu^{\prime}$, from Lemma 2.1 we see that

$$
\mathbf{i}, \mathbf{i}^{\prime} \in\left(\operatorname{supp} \mu \cap \operatorname{supp} \mu^{\prime}\right) \subset \operatorname{supp} \mu .
$$

Let us show that $\# \mathbf{j} \neq m-5, m-4, m-3$ and hence $\# \mathbf{j} \geq m-2$.

If $\# \mathbf{j}=m-5$, then by $(*), \mathbf{i}=\left(\mathbf{j}, j^{\prime}, k^{\prime}, r^{\prime}, s^{\prime}\right)$ and $\mathbf{i}^{\prime}=(\mathbf{j}, j, k, r, s)$. Thus,

$$
\mu=\frac{d_{\mathbf{j}, j^{\prime}, k^{\prime}, r^{\prime}, s^{\prime}, j, k}}{d_{\mathbf{j}, j^{\prime}, k^{\prime}, r^{\prime}, s^{\prime}, j, r}}: \frac{d_{\mathbf{j}, j^{\prime}, k^{\prime}, r^{\prime}, s^{\prime}, k, s}}{d_{\mathbf{j}, j^{\prime}, k^{\prime}, r^{\prime}, s^{\prime}, r, s}} \quad \text { and } \quad \mu^{\prime}=\frac{d_{\mathbf{j}, j, k, r, s, j^{\prime}, k^{\prime}}}{d_{\mathbf{j}, j, k, r, s, j^{\prime}, r^{\prime}}}: \frac{d_{\mathbf{j}, j, k, r, s, k^{\prime}, s^{\prime}}}{d_{\mathbf{j}, j, k, r, s, r^{\prime}, s^{\prime}}} .
$$

Since $\mu / \mu^{\prime}$ is a determinant cross ratio, and determinant polynomials are irreducible (Lemma 2.1), \#(\{j, $\left.\left.k^{\prime}, r^{\prime}, s^{\prime}\right\} \cap\{j, k, r, s\}\right)>0$, i.e. $\mathbf{i} \cap \mathbf{i}^{\prime} \neq \mathbf{j}$, a contradiction.

By similar straightforward combinatorial computations, one can prove that $\# \mathbf{j} \neq$ $m-4, m-3$. This completes the proof of the first part of the lemma.

Suppose now that $\operatorname{supp} \mu \neq \operatorname{supp} \mu^{\prime}$. Since we have already proved that $m>\# \mathbf{j} \geq$ $m-2$, we need to show that $\# \mathbf{j} \neq m-2$. Suppose to the contrary that $\# \mathbf{j}=m-2$. Then, without loss of generality, we may assume that $\mu=e_{\mathbf{j}, j^{\prime} ; j, k, r, s}$ and $\mu^{\prime}=e_{\mathbf{j}, j ; j^{\prime}, k^{\prime}, r^{\prime}, s^{\prime}}$. Therefore,

$$
\mu: \mu^{\prime}=\frac{d_{\mathbf{j}, j^{\prime}, j, k} d_{\mathbf{j}, j^{\prime}, r, s} d_{\mathbf{j}, j, j^{\prime}, r^{\prime}} d_{\mathbf{j}, j, k^{\prime}, s^{\prime}}}{d_{\mathbf{j}, j, j^{\prime}, k^{\prime}} d_{\mathbf{j}, j^{\prime}, k, s} d_{\mathbf{j}, j^{\prime}, j, r} d_{\mathbf{j}, j, r^{\prime}, s^{\prime}}}
$$

Since all determinant polynomials are irreducible (Lemma 2.1), the latter quotient is a DCR if and only if $k^{\prime}=k, r^{\prime}=r$ and $s^{\prime}=s$, which may happen if and only if supp $\mu=$ supp $\mu^{\prime}$, a contradiction.

We are left with the case $\# \mathbf{j}=m-1$, that is, $\mathbf{i}=\mathbf{i}^{\prime}$. Let $L$ be as in Remark 4.2, then the restrictions $\left.\mu\right|_{L}$ and $\left.\mu^{\prime}\right|_{L}$ are usual cross ratios of the variables $p_{j}, p_{k}, p_{r}, p_{s}$ and $p_{j^{\prime}}, p_{k^{\prime}}, p_{r^{\prime}}, p_{s^{\prime}}$, respectively, and $\left(\left.\mu\right|_{L}\right):\left(\left.\mu^{\prime}\right|_{L}\right)$ is such a cross ratio as well. By Lemma 4.3. \# $\left.\{j, k, r, s\} \cap\left\{j^{\prime}, k^{\prime}, r^{\prime}, s^{\prime}\right\}\right)=3$ and the ordered set $\left\{j^{\prime}, k^{\prime}, r^{\prime}, s^{\prime}\right\}$ is obtained by replacing one of the indices in the ordered set $\{j, k, r, s\}$ with some index $t$, where $t \neq$ $j, k, r, s$ (up to a Kleinian permutation). Consequently, \#( $\left.\operatorname{supp} \mu \cap \operatorname{supp} \mu^{\prime}\right)=\# \mathbf{i}+3=$ $m+2$ and $\mu^{\prime}$ is one of the functions $e_{\mathbf{i} ; j, k, r, t}, e_{\mathbf{i} ; k, j, s, t}, e_{\mathbf{i} ; r, s, j, t}, e_{\mathbf{i} ; s, r, k, t}$.

\section{1. $\mathbf{S}(n)$-action in $L_{\Delta}\left(\mathcal{E}^{n}\right)$}

The $\mathbf{S}(n)$-action in $\mathcal{E}^{n}$ induces an $\mathbf{S}(n)$-action on the set $L\left(\mathcal{E}^{n}\right)$ of all non-constant holomorphic functions $\mathcal{E}^{n} \rightarrow \mathbb{C} \backslash\{0,1\}$. Of course, this is the action on the set of all determinant cross ratios that we dealt with in Lemma 2.14, thus, it is transitive. If $\mu, v \in L\left(\mathcal{E}^{n}\right)$ and $\sigma \in \mathbf{S}(n)$, then the relations $\mu \mid v$ and $(\sigma \mu) \mid(\sigma v)$ are equivalent. Hence the above action induces a simplicial dimension preserving $\mathbf{S}(n)$-action on $L_{\Delta}\left(\mathcal{E}^{n}\right)$, i.e. $\mathbf{S}(n)$ acts on the sets of all simplices of any fixed dimension. Here we prove that on the set of all simplices of any positive dimension this action has two orbits.

We use the notation $(i, j)$ for the transposition of elements $i, j \in\{1, \ldots, n\}$. 
Definition 4.5. For any $s=m+3, \ldots, n$, set $I_{s}=(1, \ldots, m+2, s)$. The $t$-simplex

$$
\nabla_{1}^{t}=\left\{e_{I_{m+3}} ; e_{I_{m+4}} ; \ldots ; e_{I_{m+3+t}}\right\}
$$

is called the normal $t$-simplex of the first type; such simplices do exist for $0 \leq t \leq$ $n-m-3$. We say that a $t$-simplex is of the first type if it belongs to the $\mathbf{S}(n)$-orbit of $\nabla_{1}^{t}$. The $t$-simplex

$$
\nabla_{2}^{t}=\left\{(m-t, m) e_{I_{m+3}} ;(m-t+1, m) e_{I_{m+3}} ; \ldots ;(m-1, m) e_{I_{m+3}} ; e_{I_{m+3}}\right\}
$$

is called the normal $t$-simplex of the second type; such simplices exist for $0 \leq t \leq m-1$. We say that a $t$-simplex is of the second type if it belongs to the $\mathbf{S}(n)$-orbit of $\nabla_{2}^{t}$. Notice that for $t>0$ the simplices $\nabla_{1}^{t}$ and $\nabla_{2}^{t}$ belong to different orbits.

Remark 4.6. If $\Delta_{1}=\left\{\mu_{0}, \ldots, \mu_{t}\right\}$ and $\Delta_{2}=\left\{v_{0}, \ldots, v_{t}\right\}$ are of the same type and the corresponding sets of functions $\mu$ and $\nu$ both involve only the vector variables $q_{i_{1}}, \ldots, q_{i_{r}}$, then $\Delta_{1}$ may be carried to $\Delta_{2}$ by $\sigma \in \mathbf{S}\left(\left\{i_{1}, \ldots, i_{r}\right\}\right) \subset \mathbf{S}(n)$.

The following lemma shows that any $t$-simplex, $t>0$, is of the first or of the second type. Notice that, by Theorem 3.3 , any vertex of $L_{\Delta}\left(\mathcal{E}^{n}\right)$ is a DCR.

Lemma 4.7. Let $X$ be either $\mathbb{C}^{m}$ or $\mathbb{C P}^{m}$ and $n>m+2$.

(a) Let $\Delta=\left\{\mu_{s}\right\}_{s=1}^{l+1} \in L_{\Delta}\left(\mathcal{E}^{n}\right)$ be an l-dimensional simplex and let $\operatorname{supp} \mu_{1}=\cdots=$ $\operatorname{supp} \mu_{l+1}$. Then \# $\left(\operatorname{supp}_{\mathrm{ess}} \mu_{s} \cap \operatorname{supp}_{\mathrm{ess}} \mu_{t}\right)=m-2$ for all $t \neq s$ and $l \leq m-1$. Moreover, $\Delta$ is of the second type.

(b) Let $\Delta=\left\{\mu_{s}\right\}_{s=1}^{l+1} \in L_{\Delta}\left(\mathcal{E}^{n}\right)$ be an l-dimensional simplex. If $\operatorname{supp} \mu_{s_{0}} \neq \operatorname{supp} \mu_{t_{0}}$ for some $s_{0} \neq t_{0}$, then $\operatorname{supp} \mu_{s} \neq \operatorname{supp} \mu_{t}$ and $\operatorname{supp}_{\mathrm{ess}} \mu_{s}=\operatorname{supp}_{\mathrm{ess}} \mu_{t}$ for all $s \neq t$. Moreover, the simplex $\Delta$ is of the first type.

(c) $\operatorname{dim} L_{\Delta}\left(\mathcal{E}^{n}\right)=\max \{n-(m+3), m-1\}$.

Proof. (a) Suppose, to the contrary, that \# $\left(\operatorname{supp}_{\mathrm{ess}} \mu_{s} \cap \operatorname{supp}_{\mathrm{ess}} \mu_{t}\right) \neq m-2$ for some $s \neq t$. By Lemma 4.4 this means that \# $\left(\operatorname{supp}_{\mathrm{ess}} \mu_{s} \cap \operatorname{supp}_{\mathrm{ess}} \mu_{t}\right)>m-2$. Since \# $\operatorname{supp}_{\mathrm{ess}} \mu_{s}=$ \# $\operatorname{supp}_{\mathrm{ess}} \mu_{t}=m-1$, we have $\operatorname{supp}_{\mathrm{ess}} \mu_{s}=\operatorname{supp}_{\mathrm{ess}} \mu_{t}$; endow the latter set with some order and denote it by $\mathbf{i}=\left(i_{1}, \ldots, i_{m-1}\right)$. Let $L$ be as in Remark 4.2. Then $\left.\mu_{S}\right|_{L}$ and $\left.\mu_{t}\right|_{L}$ are usual cross ratios of the variables $p_{j}, p_{k}, p_{s}, p_{t}$ and $p_{j^{\prime}}, p_{k^{\prime}}, p_{s^{\prime}}, p_{t^{\prime}}$, respec-

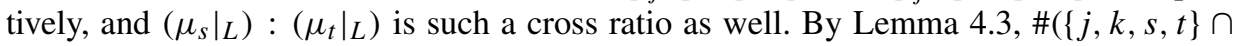
$\left.\left\{j^{\prime}, k^{\prime}, s^{\prime}, t^{\prime}\right\}\right)=3$. Consequently, \# $\left(\operatorname{supp} \mu_{s} \cap \operatorname{supp} \mu_{t}\right)=\# \operatorname{supp}_{\mathrm{ess}} \mu_{s}+3=m+2$. Since \# $\operatorname{supp} \mu_{s}=\# \operatorname{supp} \mu_{t}=m+3$, it follows that supp $\mu_{s} \neq \operatorname{supp} \mu_{t}$, a contradiction. Hence, for $s \neq t$ we have \#( $\left.\operatorname{supp}_{\mathrm{ess}} \mu_{s} \cap \operatorname{supp}_{\mathrm{ess}} \mu_{t}\right)=m-2$. Now let us show that $l \leq m-1$.

Let $\mathbf{j}=\left(i_{1}, \ldots, i_{m-2}\right)=\operatorname{supp}_{\mathrm{ess}} \mu_{1} \cap \operatorname{supp}_{\mathrm{ess}} \mu_{2}$. Then $\mu_{1}=e_{\mathbf{j}, i ; j, k, r, s}$ with certain $i, j, k, r, s$. By Lemma 2.1 and a straightforward computation, one can show that $\mu_{2} \in D=\left\{e_{\mathbf{j}, j ; i, k, r, s}, e_{\mathbf{j}, k ; j, i, r, s}, e_{\mathbf{j}, r ; j, k, i, s}, e_{\mathbf{j}, s ; j, k, r, i}\right\}$; the latter set contains no pair of functions $\left\{v, v^{\prime}\right\}$ that are vertices of the same simplex.

If $m=2$, then $\mathbf{j}=\emptyset$ and we must have $l \leq 1$, for otherwise it is easy to show that $\mu_{3} \in D$ and $\mu_{2}$ could not be a proper divisor of $\mu_{3}$. Assume now that $m>2$. 
Then \# $\left(\operatorname{supp}_{\mathrm{ess}} \mu_{1} \cap \operatorname{supp}_{\mathrm{ess}} \mu_{2} \cap \operatorname{supp}_{\mathrm{ess}} \mu_{t}\right)<m-2$ for any $t>2$, since the equality

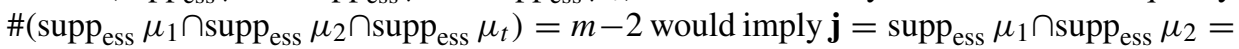
$\operatorname{supp}_{\mathrm{ess}} \mu_{1} \cap \operatorname{supp}_{\mathrm{ess}} \mu_{t}$ and $\mu_{t} \in D$, which is impossible. Since the intersection of any two of the three $(m-1)$-point sets supp ${ }_{\text {ess }} \mu_{1}$, supp $_{\text {ess }} \mu_{2}$ and supp ess $\mu_{t}$ consists of $m-2$ points, the intersection of all of them contains at least $m-3$ points. Thus, \#( $\operatorname{supp}_{\text {ess }} \mu_{1} \cap$ supp $\left._{\text {ess }} \mu_{2} \cap \operatorname{supp}_{\mathrm{ess}} \mu_{t}\right)=m-3$.

Furthermore, for any $t>2$, there is a unique $i^{\prime} \in \operatorname{supp}_{\mathrm{ess}} \mu_{1} \cap \operatorname{supp}_{\mathrm{ess}} \mu_{2}$ such that $i^{\prime} \notin \operatorname{supp}_{\text {ess }} \mu_{t}$. We shall show that $\mu_{t}$ is uniquely determined by $i^{\prime}$.

Set $\mathbf{i}=\left(i_{1}, \ldots, i_{m-3}\right)=\operatorname{supp}_{\mathrm{ess}} \mu_{1} \cap \operatorname{supp}_{\mathrm{ess}} \mu_{2} \cap \operatorname{supp}_{\mathrm{ess}} \mu_{t}$ and $\mu_{1}=e_{\mathbf{i}, i, i^{\prime} ; j, k, r, s}$. By Lemma 2.1 and a straightforward computation, one can show that $\mu_{2}$ belongs to the set $S=\left\{e_{\mathbf{i}, i^{\prime}, j ; i, k, r, s}, e_{\mathbf{i}, i^{\prime}, k ; j, i, r, s}, e_{\mathbf{i}, i^{\prime}, r ; j, k, i, s}, e_{\mathbf{i}, i^{\prime}, s ; j, k, r, i}\right\}$. Similarly, $\mu_{t} \in T=$ $\left\{e_{\mathbf{i}, i, j ; i^{\prime}, k, r, s}, e_{\mathbf{i}, i, k ; j, i^{\prime}, r, s}, e_{\mathbf{i}, i, r ; j, k, i^{\prime}, s}, e_{\mathbf{i}, i, s ; j, k, r, i^{\prime}}\right\}$. Neither $S$ nor $T$ contains a pair of functions $\left\{v, v^{\prime}\right\}$ such that $v \mid v^{\prime}$. Since \#( $\left.\operatorname{supp}_{\text {ess }} \mu_{2} \cap \operatorname{supp}_{\mathrm{ess}} \mu_{t}\right)=m-2$, for every $v \in S$ there is only one $v^{\prime} \in T$ such that $v \mid v^{\prime}$; this shows that $\mu_{t}$ is uniquely determined by $i^{\prime}$. It follows that $l=\operatorname{dim} \Delta \leq m-1$.

Finally, in view of the above facts and the transitivity of the $\mathbf{S}(n)$-action on 0 -simplices (see Lemma 2.14), the last statement of part (a) is obvious.

(b) By Lemma 4.4 for $l<2$ the statement is obvious. Suppose that $l \geq 2$. By Lemma 4.4 $\operatorname{supp}_{\mathrm{ess}} \mu_{s_{0}}=\operatorname{supp}_{\mathrm{ess}} \mu_{t_{0}}$. Assume that supp $\mu_{\tilde{s}}=\operatorname{supp} \mu_{\tilde{t}}$ for some $\tilde{s} \neq \tilde{t}$. Then, without loss of generality, we may assume $\operatorname{supp} \mu_{\tilde{s}}=\operatorname{supp} \mu_{\tilde{t}} \neq \operatorname{supp} \mu_{s_{0}}$. By Lemma 4.4 this implies that supp ${ }_{\mathrm{ess}} \mu_{\tilde{s}}=\operatorname{supp}_{\mathrm{ess}} \mu_{s}$ and $\operatorname{supp}_{\mathrm{ess}} \mu_{\tilde{t}}=\operatorname{supp}_{\mathrm{ess}} \mu_{s}$; that is, $\operatorname{supp}_{\mathrm{ess}} \mu_{\tilde{s}}=\operatorname{supp}_{\mathrm{ess}} \mu_{\tilde{t}}$, which contradicts part (a) of the lemma. Thus, supp $\operatorname{ess}_{1} \mu_{1}=$ $\cdots=\operatorname{supp}_{\mathrm{ess}} \mu_{l+1}$.

Let us prove that $\Delta$ is of the first type. Set $\mathbf{i}=\operatorname{supp}_{\mathrm{ess}} \mu_{1}=\cdots=\operatorname{supp}_{\mathrm{ess}} \mu_{l+1}$, $\mu_{1}=e_{\mathbf{i} ; j, k, r, s}$. By Lemma 4.4. \# $\left(\operatorname{supp} \mu_{1} \cap \operatorname{supp} \mu_{2}\right)=m+2$. There is a unique index $t \in \operatorname{supp} \mu_{2}$ such that $t \notin \operatorname{supp} \mu_{1}$. Since $\mu_{1} \mid \mu_{2}$, Lemma 4.4 shows that $\mu_{2} \in D=$ $\left\{e_{\mathbf{i}, j, k, r, t}, e_{\mathbf{i} ; k, j, s, t}, e_{\mathbf{i} ; r, s, j, t}, e_{\mathbf{i} ; s, r, k, t}\right\}$. After a Kleinian permutation of $j, k, r$ and $s$ in $\mu_{1}$, which never changes such a function, and an appropriate renaming of the indices in both $\mu_{1}$ and $\mu_{2}$, we may assume that $\mu_{1}=e_{\mathbf{i} ; j, k, r, s}$ and $\mu_{2}=e_{\mathbf{i} ; j, k, r, t}$.

Let $p>2$; let us prove that $\operatorname{supp} \mu_{1} \cap \operatorname{supp} \mu_{2}=\operatorname{supp} \mu_{1} \cap \operatorname{supp} \mu_{p}=\operatorname{supp} \mu_{2} \cap$ $\operatorname{supp} \mu_{p}$. First, we shall prove that $t \notin \operatorname{supp} \mu_{p}$. Suppose, on the contrary, that $t \in$ supp $\mu_{p}$. Since $\mu_{1} \mid \mu_{p}$, by Lemma 4.4, $\mu_{p} \in D$. But $D$ contains no pair of DCRs which are proper divisors of each other, which contradicts $\mu_{2} \mid \mu_{p}$. Similarly, $s \notin \operatorname{supp} \mu_{p}$. Simple combinatorics shows that $\operatorname{supp} \mu_{1} \cap \operatorname{supp} \mu_{2}=\operatorname{supp} \mu_{1} \cap \operatorname{supp} \mu_{p}=\operatorname{supp} \mu_{2} \cap$ $\operatorname{supp} \mu_{p}$. Thus, there is a unique $t_{p} \in \operatorname{supp} \mu_{p}$ such that $t_{p} \notin \operatorname{supp} \mu_{1}$. Since $\mu_{1} \mid \mu_{p}$, by Lemma 4.4 $\mu_{p} \in\left\{e_{\mathbf{i} ; j, k, r, t_{p}}, e_{\mathbf{i} ; k, j, s, t_{p}}, e_{\mathbf{i} ; r, s, j, t_{p}}, e_{\mathbf{i} ; s, r, k, t_{p}}\right\}$. Since $s \notin \operatorname{supp} \mu_{p}$, we have $\mu_{p}=e_{\mathbf{i} ; j, k, r, t_{p}}$. The group $\mathbf{S}(n)$ is $n$ times transitive on the set $\{1, \ldots, n\}$, thus, in view of Lemma 2.14, there is $\sigma \in \mathbf{S}(n)$ such that $\left\{\sigma \mu_{1}, \ldots, \sigma \mu_{l+1}\right\}=\nabla_{1}^{l}$. This completes the proof of $(\mathrm{b})$.

(c) Since the $\mathbf{S}(n)$-orbit of a simplex contains a normal simplex (see (a) and (b)), $\operatorname{dim} L_{\Delta}\left(\mathcal{E}^{n}\right)=\max \left\{\operatorname{dim} \nabla_{1}^{t}, \operatorname{dim} \nabla_{2}^{t}\right\}=\max \{n-(m+3), m-1\}$.

Lemma 4.8. Let $n>m+3$. The stabilizer $\operatorname{St}_{\mathbf{S}(n)}\left(\nabla_{1}^{n-m-3}\right)$ of the simplex $\nabla_{1}^{n-m-3}$ in the group $\mathbf{S}(n)$ coincides with the subgroup $\mathbf{S}(m-1)=\mathbf{S}(\{1, \ldots, m-1\}) \subset \mathbf{S}(n)$. 
Proof. Clearly $\mathbf{S}(m-1) \subset \operatorname{St}_{\mathbf{S}(n)}\left(\nabla_{1}^{n-m-3}\right)$. Let $\sigma \in \operatorname{St}_{\mathbf{S}(n)}\left(\nabla_{1}^{n-m-3}\right)$. Then

$$
\begin{aligned}
e_{\{1, \ldots, m-1\} ; m, m+1, m+2, m+3} & =\sigma e_{\{1, \ldots, m-1\} ; m, m+1, m+2, m+3} \\
& =e_{\{\sigma(1), \ldots, \sigma(m-1)\} ; \sigma(m), \sigma(m+1), \sigma(m+2), \sigma(m+3)},
\end{aligned}
$$

hence $\sigma$ is a disjoint product $\sigma=\phi \psi \theta$, where $\phi \in \mathbf{S}(m-1), \psi$ is one of the four Kleinian permutations Id, $(m, m+1)(m+2, m+3),(m, m+2)(m+1, m+3),(m, m+3)$ $\cdot(m+1, m+2)$ and $\theta \in \mathbf{S}(\{m+4, \ldots, n\})$ (cf. Remark 2.13). For any $t>m+3$ we have

$$
\begin{aligned}
e_{\{1, \ldots, m-1\} ; m, m+1, m+2, t} & =\phi \psi \theta e_{\{1, \ldots, m-1\} ; m, m+1, m+2, t} \\
& =e_{\{\phi(1), \ldots, \phi(m-1)\} ; \psi(m), \psi(m+1), \psi(m+2), \theta(t)} \\
& =e_{\{1, \ldots, m-1\} ; \psi(m), \psi(m+1), \psi(m+2), \theta(t) .}
\end{aligned}
$$

Consequently, $\psi=\mathrm{Id}$ and $\theta(t)=t$; since the latter is true for any $t>m+3$, we see that $\theta=\mathrm{Id}$ and $\sigma=\phi \in \mathbf{S}(m-1)$.

Lemma 4.9. The stabilizer $\operatorname{St}_{\mathbf{S}(n)}\left(\nabla_{2}^{m-1}\right)$ of the ordered simplex $\nabla_{2}^{m-1}$ in the group $\mathbf{S}(n)$ coincides with the subgroup $\mathbf{S}(\{m+4, \ldots, n\}) \subset \mathbf{S}(n)$.

Proof. Of course, any element of $\mathbf{S}(\{m+4, \ldots, n\})$ does not change $\nabla_{2}^{m-1}$. Let $\sigma \in$ $\operatorname{St}_{\mathbf{S}(n)}\left(\nabla_{2}^{m-1}\right)$. Define $I=(1, \ldots, m+3)$. Then $\nabla_{2}^{m-1}=\left\{(i, m) e_{I}\right\}_{i=1}^{m}$, where, as usual, $(i, t)$ denotes the transposition of two indices $i, t$; furthermore, $(i, m) e_{I}=\sigma(i, m) e_{I}$. For $i=m$ this means that

$$
e_{\{1, \ldots, m-1\} ; m, m+1, m+2, m+3}=e_{\{\sigma(1), \ldots, \sigma(m-1)\} ; \sigma(m), \sigma(m+1), \sigma(m+2), \sigma(m+3)}
$$

and hence $\sigma$ is a disjoint product $\sigma=\theta \phi \psi$, where $\theta \in \mathbf{S}(m-1), \phi$ is one of the four Kleinian permutations Id, $(m, m+1)(m+2, m+3),(m, m+2)(m+1, m+3)$, $(m, m+3)(m+1, m+2)$, and $\psi \in \mathbf{S}(\{m+4, \ldots, n\})$ (cf. Remark 2.13). For any $i=1, \ldots, m-1$,

$$
\begin{aligned}
e_{\{1, \ldots, i-1, m, i+1, \ldots, m-1\} ; i, m+1, m+2, m+3}=(i, m) e_{\{1, \ldots, m-1\} ; m, m+1, m+2, m+3} & \\
& =\sigma(i, m) e_{I}=\theta \phi \psi(i, m) e_{I}=\theta \phi \psi(i, m) e_{\{1, \ldots, m-1\} ; m, m+1, m+2, m+3} \\
& =e_{\{\theta(1), \ldots, \theta(i-1), \phi(m), \theta(i+1), \ldots, \theta(m-1)\} ; \theta(i), \phi(m+1), \phi(m+2), \phi(m+3) .}
\end{aligned}
$$

The last equality is satisfied if and only if $\phi=\operatorname{Id}$ and $\theta(i)=i$; since the latter is true for any $i=1, \ldots, m-1$, we see that $\theta=\operatorname{Id}$ and $\sigma=\psi \in \mathbf{S}(\{m+4, \ldots, n\})$.

\subsection{Maps of $L_{\Delta}\left(\mathcal{E}^{n}(X, g p)\right)$ induced by holomorphic self-maps}

Here we show that an equivariant endomorphism $f: \mathcal{E}^{n} \rightarrow \mathcal{E}^{n}$ induces a simplicial automorphism $f^{*}$ of $L_{\Delta}\left(\mathcal{E}^{n}\right)$. Then we prove that, for sufficiently large $n$, certain $m$ simplices are, up to a permutation of $q_{1}, \ldots, q_{n}$, fixed points of $f^{*}$.

Since the proof of the following lemma is identical to the proof of Lemma 6.1 and Corollary 6.2 from [6], we shall skip it. 
Lemma 4.10. Let $X=\mathbb{C P}^{m}$ or $\mathbb{C}^{m}$. Let $f: \mathcal{E}^{n}(X, g p) \rightarrow \mathcal{E}^{n}(X, g p)$ be an equivariant holomorphic map. If $\lambda \in L\left(\mathcal{E}^{n}(X, g p)\right)$ then $\lambda \circ f \in L\left(\mathcal{E}^{n}(X, g p)\right)$.

Corollary 4.11. Let $X=\mathbb{C P}^{m}$ or $\mathbb{C}^{m}$ and $n>m+2$. Any equivariant endomorphism $f$ of $\mathcal{E}^{n}(X, g p)$ induces a simplicial map $f^{*}$ whose restriction to each $\Delta \in L_{\Delta}\left(\mathcal{E}^{n}(X, g p)\right)$ is injective; hence $f^{*}$ preserves the dimension of any simplex.

Proof. By Lemma 4.10, for any $\lambda \in L\left(\mathcal{E}^{n}(X, g p)\right)$ we have $\lambda \circ f \in L\left(\mathcal{E}^{n}(X, g p)\right)$. Thus $f$ induces a map $f^{*}: L\left(\mathcal{E}^{n}(X, g p)\right) \ni \lambda \mapsto f^{*}(\lambda)=\lambda \circ f \in L\left(\mathcal{E}^{n}(X, g p)\right)$. If $\lambda, \mu, v \in L\left(\mathcal{E}^{n}(X, g p)\right)$ and $\lambda=\mu / v$ then $f^{*}(\mu) / f^{*}(v)=f^{*}(\mu / v)=f^{*}(\lambda)$. Hence $f^{*}$ is a simplicial map, its restriction to each simplex $\Delta \in L_{\Delta}\left(\mathcal{E}^{n}(X, g p)\right)$ is injective, and the dimension of a simplex does not change under this transformation.

Lemma 4.12. Let $X=\mathbb{C P}^{m}$ or $\mathbb{C}^{m}, n \geq m+3$ and $n \neq 2 m+2$. For any equivariant endomorphism $f$ of $\mathcal{E}^{n}(X, g p)$, the induced simplicial map $f^{*}$ is an automorphism of the complex $L_{\Delta}\left(\mathcal{E}^{n}\right)$, which preserves the type of simplices.

Proof. The set $L\left(\mathcal{E}^{n}\right)$ of all vertices of $L_{\Delta}\left(\mathcal{E}^{n}\right)$ is finite. Hence, to prove that the simplicial map $f^{*}$ is an automorphism of the complex $L_{\Delta}\left(\mathcal{E}^{n}\right)$, it suffices to show that the map $f^{*}: L\left(\mathcal{E}^{n}\right) \rightarrow L\left(\mathcal{E}^{n}\right)$ is surjective. Let $\alpha$ be the automorphism of $\mathbf{S}(n)$ associated to our equivariant endomorphism $f$ so that $f(\sigma q)=\alpha(\sigma) f(q)$ and $f^{*}(\sigma \mu)=\alpha^{-1}(\sigma)\left[f^{*}(\mu)\right]$ for all $\sigma \in \mathbf{S}(n)$ and $\mu \in L\left(\mathcal{E}^{n}\right)$. Let $\mu \in L\left(\mathcal{E}^{n}\right)$ and $v=f^{*}(\mu)$. By Lemma 2.14 . there is $\sigma \in \mathbf{S}(n)$ such that $\sigma v=\mu$. Set $\lambda=\alpha(\sigma) \mu$. Then $\mu=\sigma v=\sigma\left(f^{*}(\mu)\right)=$ $f^{*}(\alpha(\sigma) \mu)=f^{*}(\lambda)$, which proves that $f^{*}$ is surjective and therefore bijective. Hence, $f^{*}$ is a simplicial automorphism of $L_{\Delta}\left(\mathcal{E}^{n}\right)$.

Let us now prove that $f^{*}$ preserves the type of simplices. First assume that $n>$ $2 m+2$. We start with the normal simplex $\nabla_{1}^{n-m-3}$ and its faces. Since $f^{*}$ preserves dimension, $\operatorname{dim} f^{*}\left(\nabla_{1}^{n-m-3}\right)=n-m-3>m-1$ and Lemma 4.7 (a, b) shows that the simplex $f^{*}\left(\nabla_{1}^{n-m-3}\right)$ is of the first type. Any normal simplex of the first type $\nabla_{1}^{l}$ is a face of $\nabla_{1}^{n-m-3}$, and any face of a simplex of the first type is also a simplex of the first type (see Definition 4.5 . Since $f^{*}\left(\nabla_{1}^{l}\right)$ is a face of the simplex $f^{*}\left(\nabla_{1}^{n-m-3}\right)$ which is of the first type, $f^{*}\left(\nabla_{1}^{\prime}\right)$ is of the first type. Now let $\Delta \in L_{\Delta}\left(\mathcal{E}^{n}\right)$ be any $l$ simplex of the first type. It follows from Definition 4.5 that there is $\sigma \in \mathbf{S}(n)$ such that $\sigma \nabla_{1}^{l}=\Delta$. Therefore the simplex $f^{*}(\Delta)=f^{*}\left(\sigma \nabla_{1}^{l}\right)=\alpha^{-1}(\sigma) f^{*}\left(\nabla_{1}^{l}\right)$ is of the first type. Thus, $f^{*}$ carries simplices of the first type to simplices of the first type. Since the simplicial map $f^{*}$ is an automorphism of the finite complex $L_{\Delta}\left(\mathcal{E}^{n}\right), f^{*}$ is bijective on the set of all simplices of positive dimension. By Lemma 4.7 (a, b) and Definition 4.5, the latter set is a disjoint union of two of its subsets consisting of all simplices of the first and second type, respectively. Therefore, it follows from what was proved above that $f^{*}$ carries simplices of the second type to simplices of the second type. This completes the proof for $n>2 m+2$.

When $n<2 m+2$, we consider the normal simplex $\nabla_{2}^{m-1}$ and its faces. Using a similar argument, we see that $f^{*}$ carries simplices of the second type to simplices of the same type. This implies that $f^{*}$ preserves the type of all simplices, which completes the proof. 
Remark 4.13. Let $\alpha$ be the automorphism of $\mathbf{S}(n)$ associated to an equivariant endomorphism $f$ of $\mathcal{E}^{n}$. For any $\rho \in \mathbf{S}(n)$ and any function $\lambda$ on $\mathcal{E}^{n}$ we have

$$
\left.(\alpha(\rho) f)^{*}(\lambda)=\lambda \circ[\alpha(\rho) f)\right]=\lambda \circ f \circ \rho=\rho^{-1}[\lambda \circ f]=\rho^{-1}\left[f^{*}(\lambda)\right],
$$

where $\rho^{-1}[\lambda \circ f]$ is the result of the action of the permutation $\rho^{-1}$ on the function $\lambda \circ f=$ $f^{*}(\lambda)$. Changing $\rho$ to $\rho^{-1}$, we obtain

$$
\left.\rho\left[f^{*}(\lambda)\right]=\left(\alpha\left(\rho^{-1}\right) f\right)^{*}(\lambda)=\lambda \circ\left[\alpha\left(\rho^{-1}\right) f\right)\right]=(\alpha(\rho) \lambda) \circ f=f^{*}(\alpha(\rho) \lambda) .
$$

The following result plays an important part in the proof of Theorem 1.3 .

Theorem 4.14. Let $X=\mathbb{C P}^{m}$ or $\mathbb{C}^{m}, n \geq m+3$ and $n \neq 2 m+2$. For any equivariant holomorphic self-map $f$ of $\mathcal{E}^{n}(X, g p)$ there is $\rho \in \mathbf{S}(n)$ such that $(\rho f)^{*}\left(e_{\mathbf{m}(\hat{r}) ; r, m+1, m+2, s}\right)$ $=e_{\mathbf{m}(\hat{r}) ; r, m+1, m+2, s}$ for any $r \in\{1, \ldots, m\}$ and $s \in\{m+3, \ldots, n\}$. In other words, the map $(\rho f)^{*}$ is identical on each of the simplices $\left\{e_{\mathbf{m}(\hat{1}) ; 1, m+1, m+2, s}\right\}_{s=m+3}^{n}, \ldots$, $\left\{e_{\mathbf{m}(\hat{m}) ; m, m+1, m+2, s}\right\}_{s=m+3}^{n}$.

Proof. First assume that $n>m+3$. To simplify the notation, for any $s=m+3, \ldots, n$, set $I_{s}=(1, \ldots, m+2, s)$; notice that $I_{s}$ is the support of $e_{\widehat{\mathbf{m}} ; m, m+1, m+2, s}$. Notice also that the last simplex in the above list, namely

$$
\left\{e_{I_{s}}\right\}_{s=m+3}^{n}=\left\{e_{\mathbf{m}(\hat{m}) ; m, m+1, m+2, s}\right\}_{s=m+3}^{n}=\left\{e_{\widehat{\mathbf{m}} ; m, m+1, m+2, s}\right\}_{s=m+3}^{n}=\nabla_{1}^{n-m-3},
$$

is the normal $(n-m-3)$-simplex of the first type (see Definition 4.5).

By Lemma 4.12, $f^{*}$ preserves the type of simplices; hence $f^{*}\left(\nabla_{1}^{n-m-3}\right)$ is of the first type and there is $\theta \in \mathbf{S}(n)$ such that $(\theta f)^{*}\left(\nabla_{1}^{n-m-3}\right)=\nabla_{1}^{n-m-3}$. Clearly, it would be sufficient to prove the theorem for the map $\theta f$. Therefore, without loss of generality, we may assume from the very beginning that $f^{*}\left(\nabla_{1}^{n-m-3}\right)=\nabla_{1}^{n-m-3}$. Since we deal with ordered simplices, the last relation means that all vertices of $\nabla_{1}^{n-m-3}$ are fixed points of $f^{*}$, i.e.

$$
f^{*}\left(e_{I_{s}}\right)=f^{*}\left(e_{\widehat{\mathbf{m}} ; m, m+1, m+2, s}\right)=e_{\widehat{\mathbf{m}} ; m, m+1, m+2, s}=e_{I_{s}} \quad \forall s=m+3, \ldots, n .
$$

Since $f$ is equivariant, there is $\alpha \in \operatorname{Aut} \mathbf{S}(n)$ such that $f(\sigma q)=\alpha(\sigma) f(q)$ for every $\sigma \in \mathbf{S}(n)$. Consequently, for $1 \leq i<m$ and $m \leq t \leq n$ we have $f^{*}\left((i, t) \nabla_{1}^{n-m-3}\right)=$ $\alpha^{-1}((i, t)) \nabla_{1}^{n-m-3}$, where $(i, t)$ is the transposition of $i$ and $t$.

The permuted simplices

$$
\begin{aligned}
& \Delta_{s, 1}=(m+3, s) \nabla_{2}^{m-1}=\left\{(1, m) e_{I_{s}},(2, m) e_{I_{s}}, \ldots,(m-1, m) e_{I_{s}}, e_{I_{s}}\right\}, \\
& \Delta_{s, 2}=\left\{(1, m+1) e_{I_{s}},(2, m+1) e_{I_{s}}, \ldots,(m-1, m+1) e_{I_{s}}, e_{I_{s}}\right\}, \\
& \Delta_{s, 3}=\left\{(1, m+2) e_{I_{s}},(2, m+2) e_{I_{s}}, \ldots,(m-1, m+2) e_{I_{s}}, e_{I_{s}}\right\}, \\
& \Delta_{s, 4}=\left\{(1, s) e_{I_{s}},(2, s) e_{I_{s}}, \ldots,(m-1, s) e_{I_{s}}, e_{I_{s}}\right\}
\end{aligned}
$$

are simplices of the second type. By Lemma 4.12 all $f^{*}\left(\Delta_{s, \kappa}\right), \kappa=1,2,3,4$, are simplices of the second type. Since $f^{*}$ preserves vertices of $\nabla_{1}^{n-m-3}$, we have $f^{*}\left(e_{I_{s}}\right)=e_{I_{s}}$, 
and hence each of the simplices $f^{*}\left(\Delta_{s, \kappa}\right), \kappa=1,2,3,4$, contains the vertex $e_{I_{s}}$ whose essential support is $\widehat{\mathbf{m}}=(1, \ldots, m-1)$. By Lemma 4.7 (a), the essential supports of all vertices of $f^{*}\left(\Delta_{s, \kappa}\right)$ but $e_{I_{s}}$ are different from supp $\operatorname{sess}_{I_{s}}=\widehat{\mathbf{m}}$.

Claim. There is $\sigma \in \mathbf{S}(\{1, \ldots, m-1\}) \subset \mathbf{S}(n)$ such that for any $s>m+2$ the couple of simplices $f^{*}\left(\Delta_{s, 2}\right), f^{*}\left(\Delta_{s, 3}\right)$ coincides with the couple $\sigma \Delta_{s, 2}, \sigma \Delta_{s, 3}$.

Proof of Claim. We divide the proof into four steps.

Step 1. Pick some $s \geq m+3$. Let

$\Delta_{s}=f^{*}\left(\Delta_{s, 2}\right)=\left\{f^{*}\left((1, m+1) e_{I_{s}}\right), f^{*}\left((2, m+1) e_{I_{s}}\right), \ldots, f^{*}\left((m-1, m+1) e_{I_{s}}\right), e_{I_{s}}\right\}$.

Then $\Delta_{s}$ is of the second type and Lemma 4.7 (a, b) shows that supp $f^{*}\left((1, m+1) e_{I_{s}}\right)=$ $\cdots=\operatorname{supp} f^{*}\left((m-1, m+1) e_{I_{s}}\right)=\operatorname{supp} e_{I_{s}}=I_{s}$. The simplex $\Delta_{s, 2}$ is also of the second type and, according to Definition 4.5 , it may be carried to $\Delta_{s}$ by a permutation $\phi_{s} \in \mathbf{S}(n)$. The vertices of both these simplices depend only on the vector variables $q_{1}, \ldots, q_{m+2}, q_{s}$ (see Section 2 and Definition 2.8); by Remark $4.6 \phi_{s}$ may be chosen in the subgroup $\mathbf{S}\left(I_{s}\right)=\mathbf{S}(\{1, \ldots, m+2, s\}) \subset \mathbf{S}(n)$ and, by Lemma 4.9 . such a permutation is unique. In particular, $e_{I_{s}}=\phi_{s} e_{I_{s}}=\phi_{s} e_{\{1, \ldots, m-1\} ; m, m+1, m+2, s}$, i.e. $e_{I_{s}}=e_{\left\{\phi_{s}(1), \ldots, \phi_{s}(m-1)\right\} ; \phi_{s}(m), \phi_{s}(m+1), \phi_{s}(m+2), \phi_{s}(s)}$ and hence $\phi_{s}$ is a disjoint product $\phi_{s}=\sigma_{s} \theta_{s}$, where $\sigma_{s} \in \mathbf{S}(m-1)$ and $\theta_{s}$ is one of the four Kleinian permutations Id, $(m, m+1)(m+2, s),(m, m+2)(m+1, s),(m, s)(m+1, m+2)$ (cf. Remark 2.13). Consequently, for any $i=1, \ldots, m-1$ we have

$$
\theta_{s}(i, m+1) e_{I_{s}}=e_{\left\{1, \ldots, i-1, \theta_{s}(m+1), i+1, \ldots, m-1\right\} ; \theta_{s}(m), i, \theta_{s}(m+2), \theta_{s}(s)} ;
$$

the last function, in turn, must be one of the following DCRs: $(i, m+1) e_{I_{s}},(i, m) e_{I_{s}}$, $(i, s) e_{I_{s}},(i, m+2) e_{I_{s}}$. This means that $\theta_{s} \Delta_{s, 2}=\Delta_{s, j_{s}}$ for a certain $j_{s} \in\{1,2,3,4\}$ and hence

$$
\Delta_{s}=f^{*}\left(\Delta_{s, 2}\right)=\sigma_{s} \theta_{s} \Delta_{s, 2}=\sigma_{s} \Delta_{s, j_{s}} .
$$

Since $\sigma_{s} \in \mathbf{S}(m-1)$ does not touch the indices $m, m+1, \ldots, n$, relation (17) determines both $j_{s}$ and $\theta_{s}$; moreover, $j_{s}$ is uniquely determined by the value of $\theta_{s}$ on any one of the numbers $m, m+1, m+2, s$. In particular, we see that the value $\theta_{s}(m+1) \in\{m, m+1$, $m+2, s\}$ determines $j_{s}$; more precisely,

(i) the ordered couple $\left(j_{s} ; \theta_{s}(m+1)\right)$ is one of the four ordered couples $(1 ; m),(2 ; m+1)$, $(3 ; m+2),(4 ; s)$.

Step 2. Let us now show that both $\sigma_{s} \in \mathbf{S}(m-1)$ and $j_{s} \in\{1,2,3,4\}$ in (17) do not depend on $s$, and moreover $j_{s} \neq 4$.

We start with $\sigma_{s}$. Fix some $i \in\{1, \ldots, m-1\}$. By Lemma 4.12, the simplex $f^{*}\left((i, m+1) \nabla_{1}^{n-m-3}\right)$ is of the first type; the functions $f^{*}\left((i, m+1) e_{I_{m+3}}\right)$, $f^{*}\left((i, m+1) e_{I_{m+4}}\right), \ldots, f^{*}\left((i, m+1) e_{I_{n}}\right)$ are its vertices, and, by Lemma $\left.4.7 \mathrm{a}, \mathrm{b}\right)$,

$$
\operatorname{supp}_{\mathrm{ess}} f^{*}\left((i, m+1) e_{I_{m+3}}\right)=\cdots=\operatorname{supp}_{\mathrm{ess}} f^{*}\left((i, m+1) e_{I_{n}}\right) .
$$


Moreover, the function $f^{*}\left((i, m+1) e_{I_{s}}\right)$ is the $i^{\text {th }}$ vertex of the simplex $f^{*}\left(\Delta_{s, 2}\right)$; by (17), $f^{*}\left(\Delta_{s, 2}\right)=\sigma_{s} \theta_{s} \Delta_{s, 2}$ and therefore, by (16),

$$
\begin{aligned}
f^{*}\left((i, m+1) e_{I_{s}}\right) & =\sigma_{s} e_{\left\{1, \ldots, i-1, \theta_{s}(m+1), i+1, \ldots, m-1\right\} ; \theta_{s}(m), i, \theta_{s}(m+2), \theta_{s}(s)} \\
& =e_{\left\{\sigma_{s}(1), \ldots, \sigma_{s}(i-1), \theta_{s}(m+1), \sigma_{s}(i+1), \ldots, \sigma_{s}(m-1)\right\} ; \theta_{s}(m), \sigma_{s}(i), \theta_{s}(m+2), \theta_{s}(s) .}
\end{aligned}
$$

Consequently, by 18 , the set

$$
\begin{aligned}
\Sigma & :=\operatorname{supp}_{\mathrm{ess}} f^{*}\left((i, m+1) e_{I_{s}}\right) \\
& =\left\{\sigma_{s}(1), \ldots, \sigma_{s}(i-1), \theta_{s}(m+1), \sigma_{s}(i+1), \ldots, \sigma_{s}(m-1)\right\}
\end{aligned}
$$

does not depend on $s$. The only element of $\Sigma$ that is not in $\{1, \ldots, m-1\}$ is $\theta_{s}(m+1)$; hence $\theta_{s}(m+1)$ does not depend on $s$ and the same is true for the set $\Sigma^{\prime}:=\left\{\sigma_{s}(1), \ldots\right.$, $\left.\widehat{\sigma_{s}(i)}, \ldots, \sigma_{s}(m-1)\right\}$ of all elements of $\Sigma$ but $\theta_{s}(m+1)$. In fact, $\Sigma^{\prime}$ consists of all numbers $1, \ldots, m-1$ but $\sigma_{s}(i)$; thus, $\sigma_{s}(i)$ also does not depend on $s$. Since this is the case for any $i \in\{1, \ldots, m-1\}$, the permutation $\sigma:=\sigma_{s} \in \mathbf{S}(m-1)$ does not depend on $s$.

Now we turn to the index $j_{s} \in\{1,2,3,4\}$. $\theta_{s}$ is a Kleinian permutation of $m, m+1$, $m+2, s$ and we have already proved that the element $\theta_{s}(m+1) \in\{m, m+1, m+2, s\}$ does not depend on $s \in\{m+3, \ldots, n\}$; thus, $\theta_{m+3}(m+1)=\cdots=\theta_{n}(m+1) \in$ $\bigcap_{s=m+3}^{n}\{m, m+1, m+2, s\}=\{m, m+1, m+2\}$ and hence $\theta_{s}(m+1) \neq s$. By (i), this means that $j_{s} \neq 4$ and $j_{s}$ does not depend on $s$.

Thus, as a result of Steps 1 and 2, we know that

(ii) there are a permutation $\sigma \in \mathbf{S}(m-1)$ and $j \in\{1,2,3\}$ such that

$$
f^{*}\left(\Delta_{s, 2}\right)=\sigma \Delta_{s, j} \quad \text { for any } s \in\{m+3, \ldots, n\} .
$$

Step 3. In a similar way, one can show that

(ii') there is a permutation $\sigma^{\prime} \in \mathbf{S}(m-1)$ and $j^{\prime} \in\{1,2,3\}$ such that

$$
f^{*}\left(\Delta_{s, 3}\right)=\sigma^{\prime} \Delta_{s, j^{\prime}} \quad \text { for any } s \in\{m+3, \ldots, n\} .
$$

Step 4. Let us now prove that $\sigma=\sigma^{\prime}, j, j^{\prime} \neq 1$ and $j \neq j^{\prime}$. By Lemma 2.9 (b), for any $s \in\{m+3, \ldots n\}$ we have

$$
e_{I_{s}}=\left((i, m) e_{I_{s}}\right) \cdot\left((i, s) e_{I_{s}}\right)=\left((i, m+1) e_{I_{s}}\right) \cdot\left((i, m+2) e_{I_{s}}\right) .
$$

Hence, by [15],

$e_{I_{s}}=f^{*}\left(e_{I_{s}}\right)=f^{*}\left((i, m) e_{I_{s}}\right) f^{*}\left((i, s) e_{I_{s}}\right)=f^{*}\left((i, m+1) e_{I_{s}}\right) f^{*}\left((i, m+2) e_{I_{s}}\right)$.

Suppose that $j=1$. Then, by (19), $f^{*}\left((i, m+1) e_{I_{s}}\right)$ is the $i^{\text {th }}$ vertex of $f^{*}\left(\Delta_{s, 2}\right)=$ $\sigma \Delta_{s, 1}$; hence $f^{*}\left((i, m+1) e_{I_{s}}\right)=(\sigma(i), m) e_{I_{s}}$. By (22) and (21),

$$
f^{*}\left((i, m+2) e_{I_{s}}\right)=\frac{e_{I_{s}}}{f^{*}\left((i, m+1) e_{I_{s}}\right)}=\frac{e_{I_{s}}}{(\sigma(i), m) e_{I_{s}}}=(\sigma(i), s) e_{I_{s}} .
$$


By [20, this implies that $(\sigma(i), s) e_{I_{s}}$ must be the $i^{\text {th }}$ vertex of $f^{*}\left(\Delta_{s, 3}\right)=\sigma^{\prime} \Delta_{s, j^{\prime}}$; the latter shows that $j^{\prime}=4$, which contradicts (ii'). Thus $j \neq 1$. The proof of $j^{\prime} \neq 1$ is similar. Consequently, $j, j^{\prime} \in\{2,3\}$.

Now we turn to the permutations $\sigma$ and $\sigma^{\prime}$. Suppose first that $j=2$. Then, by (19), each $f^{*}\left((i, m+1) e_{I_{s}}\right), i=1, \ldots, m-1$, is a vertex of $f^{*}\left(\Delta_{s, 2}\right)=\sigma \Delta_{s, 2}$; hence $f^{*}\left((i, m+1) e_{I_{s}}\right)=(\sigma(i), m+1) e_{I_{s}}$. By (22) and (21), we obtain

$$
f^{*}\left((i, m+2) e_{I_{s}}\right)=\frac{e_{I_{s}}}{f^{*}\left((i, m+1) e_{I_{s}}\right)}=\frac{e_{I_{s}}}{(\sigma(i), m+1) e_{I_{s}}}=(\sigma(i), m+2) e_{I_{s}} .
$$

By (20), the last equality implies that the function $(\sigma(i), m+2) e_{I_{s}}$ must be the $i^{\text {th }}$ vertex of the simplex $f^{*}\left(\Delta_{s, 3}\right)=\sigma^{\prime} \Delta_{s, j^{\prime}}$. This shows that $j^{\prime}=3 \neq j$ and $\sigma^{\prime}(i)=\sigma(i)$ for any $i$ and hence $\sigma^{\prime}=\sigma$. Finally, for $j=3$, in the same way as above, we obtain $j^{\prime}=2 \neq j$ and $\sigma^{\prime}=\sigma$. This completes Step 4 and proves the Claim.

Continuing the proof of the theorem, notice that by almost the same argument as in Steps 1 and 2 above, one can show that there is a permutation $\vartheta \in \mathbf{S}(m-1) \subset \mathbf{S}(n)$ and an index $l \in\{1,2,3\}$ such that

$$
f^{*}\left(\Delta_{s, 1}\right)=\vartheta \Delta_{s, l} \quad \text { for any } s \geq m+3 .
$$

It follows from the Claim that either $l=1$ or $l \in\{2,3\}=\left\{j, j^{\prime}\right\}$, where $j$ and $j^{\prime}$ are defined by (ii) and (ii') and, according to Step 4 of the proof above, are distinct elements of the set $\{2,3\}$. Thus, we must consider the following three cases: (a) $l=1$, (b) $l=j$ and (c) $l=j^{\prime}$.

(a) In this case (23) takes the form $f^{*}\left(\Delta_{s, 1}\right)=\vartheta \Delta_{s, 1}$; therefore, using (13) for all vertices $\mu$ of the simplex $\Delta_{s, 1}$ and the permutation $\rho=\vartheta$, we obtain $(\alpha(\vartheta) f)^{*}\left(\Delta_{s, 1}\right)=$ $\vartheta^{-1}\left[f^{*}\left(\Delta_{s, 1}\right)\right]=\vartheta^{-1} \vartheta \Delta_{s, 1}=\Delta_{s, 1}$; in terms of the vertices of the ordered simplices, this means that for any $i=1, \ldots, m-1$ we have $(\alpha(\vartheta) f)^{*}\left((i, m) e_{I_{s}}\right)=(i, m) e_{I_{s}}$ and $(\alpha(\vartheta) f)^{*}\left(e_{I_{s}}\right)=e_{I_{s}}$ for all $s \geq m+3$. Since $e_{I_{m+3}}, \ldots, e_{I_{n}}$ are all the vertices of $\nabla_{1}^{n-m-3}$, we obtain $(\alpha(\vartheta) f)^{*}\left(\nabla_{1}^{n-m-3}\right)=\nabla_{1}^{n-m-3}$ and $(\alpha(\vartheta) f)^{*}\left((i, m) \nabla_{1}^{n-m-3}\right)=$ $(i, m) \nabla_{1}^{n-m-3}$ for all $i=1, \ldots, m-1$; this proves the theorem in case (a).

Let us now prove that cases (b) and (c) are impossible. Indeed, if $l=j$ then 23) and (ii) imply that $f^{*}\left(\Delta_{s, 1}\right)=\varphi\left[f^{*}\left(\Delta_{s, 2}\right)\right]$ with the permutation $\varphi=\vartheta \sigma^{-1} \in \mathbf{S}(m-1) \subset$ $\mathbf{S}(n)$ that does not depend on $s$. Therefore, using (14) for the vertices $\mu$ of the simplex $\Delta_{s, 2}$ and the permutation $\rho=\varphi$, we obtain $f^{*}\left(\Delta_{s, 1}\right)=f^{*}\left(\alpha(\varphi) \Delta_{s, 2}\right)$; since $f^{*}$ is an automorphism of the complex $L_{\Delta}\left(\mathcal{E}^{n}\right)$ (see Lemma 4.12), the latter relation implies $\Delta_{s, 1}=\alpha(\varphi) \Delta_{s, 2}$. Notice that $e_{I_{s}}$ is the very last vertex of the $(m-1)$-simplices $\Delta_{s, 1}$ and $\Delta_{s, 2}$; hence for all $s \geq m+3$ we have $e_{I_{s}}=\alpha(\varphi) e_{I_{s}}$. Since $e_{I_{m+3}}, \ldots, e_{I_{n}}$ are all the vertices of $\nabla_{1}^{n-m-3}$, we see that $\nabla_{1}^{n-m-3}=\alpha(\varphi) \nabla_{1}^{n-m-3}$. Lemma 4.8 implies that $\alpha(\varphi) \in \mathbf{S}(m-1) \subset \mathbf{S}(n)$. As $m>1$, one can see from the definition of $\Delta_{s, 1}$ and $\Delta_{s, 2}$ that $\Delta_{s, 1} \neq \psi \Delta_{s, 2}$ for any $\psi \in \mathbf{S}(m-1)$, which contradicts the equality $\Delta_{s, 1}=\alpha(\varphi) \Delta_{s, 2}$. Case (c) may be treated similarly. This completes the proof for $n>m+3$.

When $n=m+3$, the theorem asserts that there exists $\rho \in \mathbf{S}(n)$ such that $(\rho f)^{*}\left(e_{\mathbf{m}(\hat{r}) ; r, m+1, m+2, m+3}\right)=e_{\mathbf{m}(\hat{r}) ; r, m+1, m+2, m+3}$ for any $r \in\{1, \ldots, m\}$. The functions $e_{\mathbf{m}(\hat{1}) ; 1, m+1, m+2, m+3}, \ldots, e_{\mathbf{m}(\hat{m}) ; m, m+1, m+2, m+3}$ are all the vertices of the ordered 
simplex $\nabla_{2}^{m-1}$. Thus, the statement in question says that $(\rho f)^{*}\left(\nabla_{2}^{m-1}\right)=\nabla_{2}^{m-1}$ for an appropriate permutation $\rho \in \mathbf{S}(n)$. Since $f$ is equivariant, this is equivalent to the existence of $\phi \in \mathbf{S}(n)$ such that $f^{*}\left(\nabla_{2}^{m-1}\right)=\phi \nabla_{2}^{m-1}$, which means precisely that the simplex $f^{*}\left(\nabla_{2}^{m-1}\right)$ is of the second type. The last property follows from Lemma 4.12 . which completes the proof.

\section{The proof of Theorem 1.3}

Here we prove the main result of this paper. We start with the following remark which is similar to Remark 2.14 in [6].

Remark 5.1. For $n \geq m+3$, there is a non-empty Zariski open subset $U \subset \mathcal{E}^{n}(X, g p)$ such that if $A q=\sigma q$ for some $q \in U, A \in \operatorname{PSL}(\mathbb{C}, m+1)$ and $\sigma \in \mathbf{S}(n)$ then $A=\operatorname{Id}$ and $\sigma=$ Id. Indeed, Lemma 2.5 implies that for any two points $q=\left(q_{1}, \ldots, q_{n}\right)$ and $q^{\prime}=$ $\left(q_{1}^{\prime}, \ldots, q_{n}^{\prime}\right)$ in $\mathcal{E}^{n}(X, g p)$, an element $A \in \mathbf{P S L}(\mathbb{C}, m+1)$ is uniquely determined by the requirement $A q_{i}=q_{i}^{\prime}$ for all $i=1, \ldots, m+2$. Since $\mathbf{S}(n)$ is finite, it follows that the set $S$ of all points $q=\left(q_{1}, \ldots, q_{n}\right) \in \mathcal{E}^{n}(X, g p)$ such that for some $A \in \operatorname{PSL}(\mathbb{C}, m+1)$ and some non-trivial permutation $\sigma \in \mathbf{S}(n)$ (both $A$ and $\sigma$ may depend on $q$ ) the point $A q=\left(A q_{1}, \ldots, A q_{n}\right)$ coincides with the point $\sigma q=\left(q_{\sigma^{-1}(1)}, \ldots, q_{\sigma^{-1}(n)}\right)$, is a proper Zariski closed subset of $\mathcal{E}^{n}(X, g p)$. Its complement $U=\mathcal{E}^{n}(X, g p) \backslash S$ is the desired non-empty Zariski open set.

Proof of Theorem 1.3. By Theorem 4.14, there is a permutation $\rho$ such that

$$
e_{\mathbf{m}(\hat{r}) ; r, m+1, m+2, s}(\rho f(q))=e_{\mathbf{m}(\hat{r}) ; r, m+1, m+2, s}(q)
$$

for all $q \in \mathcal{E}^{n}(X, g p), s=m+3, \ldots, n$ and $r=1, \ldots, m$. Lemma 2.5 implies that there is a map $\gamma: \mathcal{E}^{n}\left(\mathbb{C P}^{m}, g p\right) \rightarrow \operatorname{PSL}(\mathbb{C}, m+1)$ such that $\gamma(q) q \in M_{m, n}$ (see Definition 2.4). Lemma 2.15 says that DCRs are $\operatorname{PSL}(\mathbb{C}, m+1)$-invariant; hence

$$
\begin{aligned}
e_{\mathbf{m}(\hat{r}) ; r, m+1, m+2, s}(\gamma(q) q) & =e_{\mathbf{m}(\hat{r}) ; r, m+1, m+2, s}(q), \\
e_{\mathbf{m}(\hat{r}) ; r, m+1, m+2, s}(\gamma(\rho f(q)) \rho f(q)) & =e_{\mathbf{m}(\hat{r}) ; r, m+1, m+2, s}(\rho f(q))
\end{aligned}
$$

for all $q \in \mathcal{E}^{n}(X, g p), s \in\{m+3, \ldots, n\}$ and $r \in\{1, \ldots, m\}$. Comparing (24) and 25) we see that

$$
e_{\mathbf{m}(\hat{r}) ; r, m+1, m+2, s}(\gamma(q) q)=e_{\mathbf{m}(\hat{r}) ; r, m+1, m+2, s}(\gamma(\rho f(q)) \rho f(q))
$$

for any $q \in \mathcal{E}^{n}(X, g p)$ and all $s \in\{m+3, \ldots, n\}$ and $r \in\{1, \ldots, m\}$. Both points $\gamma(q) q$ and $\gamma(\rho f(q))(\rho f(q))$ are in $M_{m, n}$, and Lemma 2.17 says that the functions $e_{\mathbf{m}(\hat{r}) ; r, m+1, m+2, s}$ with $s \in\{m+3, \ldots, n\}$ and $r \in\{1, \ldots, m\}$ separate points of $M_{m, n}$. Consequently, (*) implies that $\gamma(\rho f(q)) \rho f(q)=\gamma(q) q$, or, which is the same, $\rho f(q)=(\gamma(\rho f(q)))^{-1} \gamma(q) q$. Set $\tau(q)=(\gamma(\rho f(q)))^{-1} \gamma(q)$. The map $\tau: \mathcal{E}^{n}(X, g p) \ni$ $q \mapsto \tau(q) \in \operatorname{PSL}(\mathbb{C}, m+1)$ is holomorphic and $\tau(q) q=\rho f(q)$, that is, $f(q)=\sigma \tau(q) q$ for all $q \in \mathcal{E}^{n}(X, g p)$, where $\sigma=\rho^{-1} \in \mathbf{S}(n)$. 
To complete the proof, we must check that the morphism $\tau$ is $\mathbf{S}(n)$-invariant. Let $\alpha \in \operatorname{Aut} \mathbf{S}(n)$ be the automorphism associated to our equivariant map $f$. For every $\theta \in$ $\mathbf{S}(n)$ and all $q \in \mathcal{E}^{n}(X, g p)$ we have $\sigma \tau(\theta q) \theta q=f(\theta q)=\alpha(\theta) f(q)=\alpha(\theta) \sigma \tau(q) q$, which can be written as $\left[(\tau(\theta q))^{-1} \cdot \tau(q)\right] q=\sigma^{-1} \alpha\left(\theta^{-1}\right) \sigma \theta q$, where $(\tau(\theta q))^{-1} \cdot \tau(q)$ is a product in PSL $(\mathbb{C}, m+1)$. In view of Remark 5.1, this implies that $\sigma^{-1} \alpha\left(\theta^{-1}\right) \sigma \theta=\mathrm{Id}$ and $\tau(\theta q)=\tau(q)$ for all $\theta \in \mathbf{S}(n)$ and all $q$ in a non-empty Zariski open subset $U \subset$ $\mathcal{E}^{n}(X, g p)$. Since $\tau$ is continuous, the last equality holds true for all $q \in \mathcal{E}^{n}(X, g p)$; since $\theta \in \mathbf{S}(n)$ was arbitrary, this shows that the morphism $\tau: \mathcal{E}^{n}(X, g p) \rightarrow \operatorname{PSL}(\mathbb{C}, m+1)$ is $\mathbf{S}(n)$-invariant. This completes the proof.

Theorem 1.3 and Definition 1.1 imply the following corollary.

Corollary 5.2. Let $m>1, n \geq m+3$ and $n \neq 2 m+2$.

(a) Any holomorphic map $F: \mathcal{C}^{n}\left(\mathbb{C P}^{m}\right.$, gp $) \rightarrow \mathcal{C}^{n}\left(\mathbb{C P}^{m}\right.$, gp) that can be lifted to an equivariant holomorphic map $f: \mathcal{E}^{n}\left(\mathbb{C P}^{m}, g p\right) \rightarrow \mathcal{E}^{n}\left(\mathbb{C P}^{m}, g p\right)$ is tame.

(b) Any holomorphic map $F: \mathcal{C}^{n}\left(\mathbb{C}^{m}, g p\right) \rightarrow \mathcal{C}^{n}\left(\mathbb{C}^{m}, g p\right)$ that can be lifted to an equivariant holomorphic map $f: \mathcal{E}^{n}\left(\mathbb{C}^{m}, g p\right) \rightarrow \mathcal{E}^{n}\left(\mathbb{C}^{m}, g p\right)$ is quasitame.

Acknowledgments. This paper is based upon my $\mathrm{PhD}$ thesis supported by Technion. I wish to thank V. Lin who introduced me to the problem and encouraged me to work on it. I also wish to express my thanks to M. Entov and A. Vershik for stimulating discussions and to B. Shapiro for useful references.

\section{References}

[1] Barvinok, A. I.: Homological type of spaces of configurations of structurally stable type in $\mathbb{C}^{2}$. Mat. Zametki 39, 108-112 (1986) (in Russian) Zbl 0601.51022 MR 0830847

[2] Efimov, N. V.: Higher Geometry. Translated from the sixth Russian edition by P. C. Sinha, Mir, Moscow (1980) Zbl 0457.51001 MR 0607502

[3] Feler, Y.: Configuration spaces of tori. Rend. Lincei Mat. Appl. 18, 139-151 (2007) MR 2314168

[4] Lin, V. Ya.: Algebraic functions with universal discriminant manifolds. Funct. Anal. Appl. 6, 73-75 (1972) Zbl 0249.12101 MR 0296343

[5] Lin, V. Ya.: Artin braids and the groups and spaces connected with them. Itogi Nauki i Tekhniki, Algebra, Topologiya, Geometriya 17, VINITI, Moscow, 159-227 (1979) (in Russian); English transl.: J. Soviet Math. 18, 736-788 (1982) Zbl 0479.20016 MR 0584570

[6] Lin, V. Ya.: Configuration spaces of $\mathbb{C}$ and $\mathbb{C P}^{1}$ : some analytic properties. Max-Planck-Institut für Mathematik Preprint Series 2003 (98), Bonn, 80 pp. (2003); Revised electronic version arXiv:math.AG/0403120

[7] Möbius, A. F.: Der barycentrische Calcul. Verlag von Johann Ambrosius Barth, Leipzig (1827) Zbl 0355.01010 MR 0462890

[8] Moulton, V. L.: Vector braids. J. Pure Appl. Algebra 131, 245-296 (1998) Zbl 0999.20027 MR 1637019

[9] Terasoma, T.: Fundamental groups of moduli spaces of hyperplane configurations. http://gauss.ms.u-tokyo.ac.jp/paper/paper.html 
[10] Weyl, H.: The Classical Groups. Their Invariants and Representations. Princeton Univ. Press, Princeton, NJ (1946) Zbl 0020.20601 MR 0000255

[11] Zinde, V. M.: Commutants of Artin groups. Uspekhi Mat. Nauk 30, no. 5 (185), 207-208 (1975) (in Russian) Zbl 0338.20042 MR 0432767

[12] Zinde, V. M.: Analytic properties of the spaces of regular orbits of Coxeter groups of the series $B$ and $D$. Funktsional. Anal. i Prilozhen. 11, no. 1, 69-70 (1977) (in Russian) Zbl 0408.20033 MR 0481097

[13] Zinde, V. M.: Holomorphic mappings of the spaces of regular orbits of Coxeter groups of series $B$ and D. Sibirsk. Mat. Zh. 18, no. 5, 1015-1026, 1205 (1977) (in Russian) Zbl 0387.32012 MR 0460713

[14] Zinde, V. M.: Some homomorphisms of the Artin groups of the series $B_{n}$ and $D_{n}$ into groups of the same series and into symmetric groups. Uspekhi Mat. Nauk 32, no. 1 (193), 189-190 (1977) (in Russian) Zbl 0361.20056 MR 0444781

[15] Zinde, V. M.: Studies of homomorphisms of Artin groups. C. R. Math. Rep. Acad. Sci. Canada 1, 199-200 (1978/79) Zbl 0425.20031 MR 0541460 